\title{
Failure damage mechanical properties of thoracic and abdominal porcine aorta layers and related constitutive modeling: Phenomenological and microstructural approach
}

\author{
Juan A. Peña · Miguel A. Martínez • Estefanía Peña
}

Received: date / Revised version: date

\begin{abstract}
Despite increasing experimental and analytical efforts to investigate the irreversible effects of arterial tissue failure, the underlying mechanisms are still poorly understood. The goal of this study was characterize the failure properties of the intact wall and each separated layer (intima, media and adventitia) of the descending thoracic and infrarenal abdominal aorta and to test the hypothesis that the failure properties of layer-separated tissue depend on the location of the aorta. To test this hypothesis, we performed uniaxial tests to study the mechanical behavior of both intact and layer-separated porcine aortic tissue samples taken from descending thoracic and infrarenal abdominal aorta until complete failure. The fracture stress is higher in the infrarenal abdominal aorta than in the equivalent descending thoracic aorta. It was also found that the extrapolation of the elastic mechanical properties from the physiological to the supra-physiological regime for characterizing the mechanical response of the aorta would be inappropriate. Finally, we report values of constitutive parameters using phenomenological and microstructural damage models based on Continuum Damage Mechanics Theory. The phenomenological damage model gives an excellent fit to the experimental data compared to the microstructural damage model. Although the fitting
\end{abstract}

Juan A. Peña · Miguel A. Martínez · Estefanía Peña at Aragón Institute of Engineering Research (I3A). University of Zaragoza, Spain.

Juan A. Peña at Department of Management and Manufacturing Engineering. Faculty of Engineering and Architecture. University of Zaragoza. Spain.

Miguel A. Martínez · Estefanía Peña at CIBER de Bioingeniería, Biomateriales y Nanomedicina (CIBER-BBN). Spain.

Estefanía Peña. fany@unizar.es Mechanical Engineering Department. c/ Maria de Luna s/n 50018. Zaragoza. Spain. Tel.: $+34876555233$ results of the phenomenological model are better, the microstructural models can include physically motivated aspects obtained from experiments.

Keywords: Failure properties; damage mechanics; thoracic and abdominal aorta; layer separation

\section{Introduction}

Mechanical force at the tissue level leads to local stress concentrations within the tissue and, if strong enough, begins to cause damage at specific spots. In healthy tissues at physiological stress levels, healing continuously repairs such defects to maintain the structural integrity of the tissue. Despite increasing experimental and analytical efforts to investigate failure-related irreversible effects of soft biological tissue, the underlying mechanisms are still poorly understood. In general, three important damage phenomena associated with arteries may be distinguished. First, there is the dependence of the mechanical response on the previously attained maximum load level. This is very similar to the well-known Mullins effect in rubber-like materials (Alastrué et al., 2008; García et al., 2013; Peña, 2011c). Another typical phenomenon known as permanent set is characterized by residual strains after unloading (Alastrué et al., 2008; García et al., 2013). Finally, there is failure resulting from fibre rupture and matrix disruption associated with material damage and fracture (Peña, 2011a,c; Peña et al., 2009). Elastic arterial biomechanics has been intensively studied. Experimental studies have used different kinds of procedures such as inflation tests (Barra et al., 1993; Kim \& Baek, 2011; Lillie et al., 2012; Lu et al., 2003), simple uniaxial tension (Silver et al., 
2003; Sokolis, 2010; Weisbecker et al., 2012), and biaxial tests (Kamenskiy et al., 1998; Lally et al., 2004; Zeinali-Davarani et al., 2013) to determine the elastic mechanical properties of aortic tissues. However, there are few experimental analyses of damage or failure mechanics of arteries (García et al., 2013; Peña, 2011a,c; Peña et al., 2009; Pierce et al., 2015). Only Weisbecker et al. (Weisbecker et al., 2012) addresses the layer specific softening response of the human aorta. However, their study only presented the softening (Mullin's effect) response of the layers and there was no data about the failure and rupture properties. While it is well-known that aortic elastic properties vary along the aortic tree, it is generally agreed that aortic stiffness increases with increasing distance from the heart (Guo \& Kassab, 2003; Hang \& Fung, 1995; Kim et al., 2013; Peña et al., 2018, 2015). Clearly, damage mechanisms and specific injury tolerance are closely related to the individual tissue types, but collagen seems to play a universal pivotal role. Parameters such as aortic wall thickness, moisture content, and the location of the specimen along the aorta may also influence the damage response.

The constitutive response of biological tissues, and their variation with disease processes must be wellcharacterized, both, experimentally and theoretically. Damage scenarios such as device deployment processes, in vivo operating conditions, and and the surgeon's skill must be simulated accurately. Model predictions should also reflect the heterogeneity, nonlinearity, anisotropy and their dependence on the arterial location of the vessel. With all these facts to hand, the incorporation of damage in mechanical constitutive models of arteries is a prerequisite for a better understanding of clinical treatments involving supraphysiological loading.

There are several damage formulations able to describe the failure of arteries (Balzani et al., 2006; Calvo et al., 2007; Hokanson \& Yazdami, 1997; Li \& Robertson, 2009; Peña, 2011b; Peña et al., 2009; Rodríguez et al., 2006; Sáez et al., 2012; Volokh, 2007a; Wulandana \& Robertson, 2005). Balzani et al. (Balzani et al., 2006) assumed that discontinuous damage occurs mainly along the fiber direction in the arterial wall. Rodriguez et al. (Rodríguez et al., 2006) introduced a stochastic-structurally based damage model for fibrous soft tissues in general and arteries in particular. Calvo et al. (Calvo et al., 2007) proposed an uncoupled directional damage model for fibred biological soft tissues that considers different damage evolutions for the matrix and fibers which was applied to model the damage process during arterial clamping. In $\mathrm{Li}$ and Robertson (Li \& Robertson, 2009), two damage mechanisms are coupled in a multiplicative manner for cerebral arteries. Peña et al. (Peña et al., 2009) showed that continuum damage mechanics models can reproduce the softening behavior during unloading or reloading only for low dissipative effects. Ehret et al. (Ehret \& Itskov, 2009) and Peña (Peña, 2011c) presented different models that reproduce the softening behavior including all the tissue dissipative effects (including permanent set). Peña and Doblaré (Peña \& Doblare, 2009) present a very simple pseudo-elastic anisotropic model to reproduce the softening behavior exhibited in arteries without permanent set. Later, similar model was used by Weisbecker et al. (Weisbecker et al., 2012) to reproduce the layer spedific Mullin's effect of aorta. Of all of these models, only the stochastic-structurally based damage model (Rodríguez et al., 2006; Sáez et al., 2012) and Kachanov-like damage models (Calvo et al., 2007; Peña et al., 2009; Sáez et al., 2012) are able to reproduce the failure region as a result of the bond rupture and complete damage presented in arteries.

The goal of this study was to characterize the failure properties of the intact wall and each separated layer (intima, media and adventitia) of the descending thoracic and infrarenal abdominal aorta and to test the hypothesis that the failure properties of layerseparated thoracic arteries differ depending on arterial location in the aorta. To test this hypothesis, we performed uniaxial tests to study the mechanical behavior of both intact and layer-separated porcine aortic tissue samples taken from descending thoracic and infrarenal abdominal aorta until complete failure. For its simplicity and applicability, a phenomenological Continuum Damage based damage model (Calvo et al., 2007) and its extension to a microstructural damage model (Sáez et al., 2012) are considered in this work to reproduce the experimental data. Finally, we also provided and compared the damage parameters using both damage models with a particularized damage function for arteries proposed in Peña (Peña, 2011b) for each layer that can be used by biomedical engineers for investigating better therapies and developing artery-specific devices.

\section{Experiments}

Seven thoracic and abdominal aortas $(\mathrm{n}=7)$ were excised postmorten from $3.5 \div 0.45$ month old female pigs, sacrificed for other animal studies after which the aorta remained intact. All animal experiments were performed in accordance with the "Principles of Laboratory Animal Care"' (86/609/EEC Norm, incorporated into Spanish legislation through the RD 
1021/2005) and approved by the Ethical Committee for Animal Research of the University of Zaragoza. The animals were anesthetized with potassium chloride and sodium thiopental, and the aortas were isolated and dissected free of perivascular tissue and preserved in ion-free PSS $(0.9 \% \mathrm{NaCl})$ at $4\left[{ }^{\circ} \mathrm{C}\right]$ until preparation the test samples.

\subsection{Mechanical tests}

Two circumferential and two longitudinal strips (approximately $5 \times 15[\mathrm{~mm}]$ ) were cut from the proximal part of the descending thoracic (DTA) and infrarenal abdominal aortas (IAA). Thus, a minimum of four circumferential and longitudinal samples were used per animal. One pair of sample strips taken from each aorta direction were tested as whole samples. In other pair of samples, the intimal layer was dissected very carefully using microsurgery instruments, and then the media was separated from the adventitia (Peña et al., 2015). The correct layer separation without damage were checked by histological images and damaged samples was discarded. The size of the samples were measured by three measurements at different locations using a contact Mitutoyo Electronic Digital caliper with a contact force of $0.5 \mathrm{~N}$ and the averaged values were considered.

Uniaxial tests of the circumferential and axial strips were carried out in a high precision drive Instron Microtester 5548 system adapted for biological tissues with a $10[\mathrm{~N}]$ load cell having a minimal resolution of $0.005[\mathrm{~N}]$ and using a non-contact Instron 2663281 video-extensometer to measure the axial strain. To avoid specimen drying, tests were performed with an ultrasonic humidifier. Previous to each test, the zero load was defined after the tissue specimen was located in the grips. The specimens were preconditioned by different loading and unloading cycles from zero to a maximum engineering stress (240 [kPa]) using a triangular waveform at a $30 \% / \mathrm{min}$ strain rate. To characterize the damage behavior, after the preconditioning the specimens were loaded until full rupture. Only the samples whose rupture was located in the middle of the sample between markers were considered for subsequent damage analysis. The Cauchy stress was computed as $\sigma_{\theta \theta, z z}=\frac{F_{\theta, z}}{t_{\theta, z} w_{\theta, z}} \lambda_{\theta, z}$, where $F$ is the load registered by the Instron machine and $t_{\theta, z}$ $w_{\theta, z}$ are the mean computed thickness and with, and $\lambda_{\theta, z}$ is the stretch in the circumferential and longitudinal directions respectively measured by the videoextensometer.

To gain some insight into the influence of the specific layer characteristics on the damage properties of thoracic and abdominal aorta, the maximum values of stress and stretch, $\sigma_{r}$ and $\lambda_{r}$ respectively, for both circumferential and longitudinal samples were analyzed.

\subsection{Histological analysis}

One part of each specimen from the DTA and IAA samples (approximately $0.5 \times 0.5 \mathrm{~cm}$ ) was used for microscopic investigation. Segments of each vessel were fixed in formaldehyde for $24 \mathrm{~h}$ and then placed in $70 \%$ alcohol. The segments were dehydrated and embedded in paraffin. The histology blocks were cross-sectioned at 5 microns and stained with Hematoxiline-eosine in order to study the general structure of the sample and to check that the samples were successfully separated..

\subsection{Statistical analysis}

A statistical analysis was performed in order to analyze possible significant variations in the damage or failure behavior of the aorta and each of its layers (intima, median and adventitia) along the position of the vessel Therefore, the maximum values of stress and stretch $\left(\sigma_{r}\right.$ and $\left.\lambda_{r}\right)$ for the DTA and IAA were compared for both circumferential and longitudinal directions for the whole aorta and each individual layer. Normal distribution of the variables was tested using the Shapiro-Wilk test. All groups were compared by means of an independent one-tailed $t$-test. In cases of non-normal distribution, the Mann-Whitney test was used. We established $p<0.05$ as the level to indicate statistical significance.

\section{Theoretical framework}

In recent years the most widely used approach for modeling anisotropy in soft tissues has been representing fiber directions by means of an invariant formulation, referred to in this work as the "Phenomenological model" (Peña, 2011b). The use of statistical distributions has recently increased and is also adopted in the present work, referred to as the "Microstructural model". Furthermore, a microsphere-based approach has been used at a micro scale level (Sáez et al., 2012).

\subsection{Phenomenological anisotropic hyperelastic damage model for vascular tissue}

In the context of continuum damage mechanics, we assume the existence of a free energy function decoupled 
into volumetric and isochoric parts where the isochoric contribution is additively decomposed into isotropic and anisotropic contributions as

$\Psi=\Psi_{v o l}(J)+\Psi_{i s o}\left(\overline{\boldsymbol{C}}, D_{m}\right)+\Psi_{a n i}\left(\overline{\boldsymbol{C}}, \boldsymbol{M}, \boldsymbol{N}, D_{f}\right)$,

where $\Psi_{v o l}(J)$ represents the free energy associated to volume changes, $J=\operatorname{det} \boldsymbol{F}$ the local volume ratio, and $\overline{\boldsymbol{C}}=\overline{\boldsymbol{F}}^{t} \cdot \overline{\boldsymbol{F}}$ the isochoric right Cauchy-Green deformation tensor $\overline{\boldsymbol{C}}=\overline{\boldsymbol{F}}^{t} \cdot \overline{\boldsymbol{F}}$ computed by the isochoric deformation gradient tensor $\overline{\boldsymbol{F}}=J^{-1 / 3} \boldsymbol{F}$ (Flory, 1961). The anisotropic response is considered by the structural tensors $\boldsymbol{M}=\boldsymbol{m} \otimes \boldsymbol{m}$ and $\boldsymbol{N}=\boldsymbol{n} \otimes \boldsymbol{n}$ that accounts the two families of fibres being embedded in the continuum defined by means of unit vectors, namely $\boldsymbol{m}$ and $\boldsymbol{n}$. Finally, $D_{m} \in[0,1]$ and $D_{f} \in[0,1]$ are monotonically increasing internal damage variables.

In terms of the strain invariants, the free energy function $\Psi$ is computed as

$$
\begin{aligned}
\Psi & =\Psi_{\text {vol }}(J)+\left[1-D_{m}\right] \Psi_{i s o}\left(\bar{I}_{1}\right) \\
& +\left[1-D_{f}\right]\left[\Psi_{a n i}\left(\bar{I}_{4}\right)+\Psi_{a n i}\left(\bar{I}_{6}\right)\right]
\end{aligned}
$$

where $\bar{I}_{4}=\overline{\boldsymbol{C}}: \boldsymbol{M}$ and $\bar{I}_{6}=\overline{\boldsymbol{C}}: \boldsymbol{N}$ are isochoric anisotropic invariants (see, e.g., Spencer, 1971). $\left[1-D_{m}\right]$ and $\left[1-D_{f}\right]$ are reduction factors that weigh the strain energy associated to the undamaged isotropic and anisotropic contributions, respectively (Calvo et al., 2007).

The strain energy associated to the undamaged isotropic contribution was assumed to be represented by the simple neo-Hookean term and the mechanical behavior associated to the undamaged fibres is assumed to correspond to the strain energy function (SEF) proposed by Gasser et al. (Gasser et al., 2006), i.e.

$$
\Psi=\mu\left[I_{1}-3\right]+\sum_{i=4,6}\left[\frac{k_{1}}{2 k_{2}}\left[\exp \left(k_{2} \hat{E}_{i}^{2}-1\right)\right]\right],
$$

where

$$
\hat{E}_{i}=\kappa I_{1}+[1-3 \kappa] I_{i}-1 \quad i=4,6
$$

and $\kappa \in[0,1 / 3]$ is a dispersion parameter; when $\kappa=0$, the model is equivalent to that published in Holzapfel et al. (Holzapfel et al., 2000), and when $\kappa=1 / 3$ it recovers an isotropic potential similar to that used in Demiray (Demiray, 1972). $\mu>0$ and $k_{1}>0$ are stresslike parameters and $k_{2}>0$ is dimensionless.

From the Clausius-Planck inequality and (2), the second Piola-Kirchhoff stress can be derived:

$$
\begin{aligned}
\mathbf{S} & =2 \frac{\partial \Psi(\mathbf{C}, \mathbf{M})}{\partial \mathbf{C}}=J p \mathbf{C}^{-1}+2 \sum_{j=1,2,4} \frac{\partial \bar{\Psi}}{\partial \bar{I}_{j}} \frac{\partial \bar{I}_{j}}{\partial \overline{\mathbf{C}}}: \frac{\partial \overline{\mathbf{C}}}{\partial \mathbf{C}} \\
& =\mathbf{S}_{v o l}+\left[1-D_{m}\right] \overline{\mathbf{S}}_{0}^{m}+\left[1-D_{f}\right] \overline{\mathbf{S}}_{0}^{f}
\end{aligned}
$$

\subsubsection{Damage criterion}

A discontinuous damage criterion (Peña et al., 2009) for matrix $(m)$ and fibers $(f)$ is defined in the strain space by the condition at any time $t$ of the loading process (Simo, 1987)

$$
\begin{aligned}
\Phi_{m, f}\left(\overline{\boldsymbol{C}}(t), \Xi_{m, f}^{*}\right) & =\sqrt{2 \Psi_{m, f}^{0}(\overline{\boldsymbol{C}}(t))}-\Xi_{m, f}^{*} \\
& =\Xi_{m, f}-\Xi_{m, f}^{*} \leq 0
\end{aligned}
$$

where $\overline{\boldsymbol{C}}(s)$ the isochoric right Cauchy-Green tensor at time $s$ and

$$
\Xi_{m, f}^{*}=\max _{s \in(-\infty, t]} \sqrt{2 \Psi_{m, f}^{0}(\bar{C}(s))}
$$

Note that $\Phi_{m, f}\left(\overline{\boldsymbol{C}}(t), \Xi_{m, f}^{*}\right)=0$ defines a damage surface in the strain space (Peña et al., 2009). The evolution of the internal damage parameters $D_{\boldsymbol{m}, \boldsymbol{f}}$ is defined by the following irreversible evolution equation

$$
\frac{\mathrm{d} D_{m, f}}{\mathrm{~d} t}= \begin{cases}h_{m, f}\left(\Xi_{m, f}, D_{m, f}\right) \dot{\Xi}_{m, f} & \text { if } \Phi=0 \\ 0 & \text { and } \boldsymbol{N}_{m, f}: \dot{\overline{\boldsymbol{C}}}>0 \\ \text { otherwise }\end{cases}
$$

where $\boldsymbol{N}_{m, f}:=\partial_{\bar{C}} \Phi_{m, f}$ is the normal to the damage surface in the strain space, $\Xi_{m, f}$ are defined at the current time $s, \dot{\Xi}_{m, f}$ and $\dot{\overline{\boldsymbol{C}}}$ the time rate of change of $\Xi_{m, f}$ and $\overline{\boldsymbol{C}}$ respectively and $\bar{h}_{m, f}\left(\Xi_{m, f}, D_{m, f}\right)$ characterize the evolution of the damage in the material. Following Peña (Peña, 2011b), the expression of the damage function is a sigmoidal type function as

$$
D_{m, f}=\frac{1}{1+\exp \left(-\alpha_{m, f}\left[\Xi_{m, f_{t}}-\gamma_{m, f}\right]\right)},
$$

where the parameter $\alpha_{m, f}$ controls the slope and $\gamma_{m, f}$ defines the value $\Xi_{m, f_{t}}$ such that $D_{m, f}\left(\Xi_{m, f_{t}}\right)=0.5$. We choose the sigmoidal function (18) as it is realvalued and differentiable, having either a non-negative or non-positive first derivative which is bell shaped and there is also a pair of horizontal asymptotes in 0 and 1 (Peña, 2011b; Sáez et al., 2012).

3.2 Microstructural anisotropic hyperelastic damage model of vascular tissue

The microsphere approach tries to capture microstructural information and transfer it into the macroscopic behavior via a homogenization scheme over the unit sphere $\mathbb{U}^{2}$. In this approach, $\mathbb{U}^{2}$ is discretized into $m$ directions $\left\{\mathbf{r}^{i}\right\}_{i=1 \ldots m}$ that are weighted by factors $\left\{w^{i}\right\}_{i=1 \ldots m}$, where $\langle\mathbf{r}\rangle \approx \sum_{i=1}^{m} w^{i} \mathbf{r}^{i}=0$ and 
$\langle\mathbf{r} \otimes \mathbf{r}\rangle \approx \sum_{i=1}^{m} w^{i} \mathbf{r}^{i} \otimes \mathbf{r}^{i}=\frac{1}{3} \mathbf{I}$. So an integral over the unit sphere $\mathbb{U}^{2}$ can be approximated by

$$
\langle(\bullet)\rangle=\frac{1}{4 \pi} \int_{\mathbb{U}^{2}}(\bullet) \mathrm{dA} \approx \sum_{i=1}^{m} w^{i}(\bullet)^{i} .
$$

The term $4 \pi$ is a normalization factor, the result of the surface integral $\int_{0}^{\theta} \int_{0}^{\phi} \sin (\theta) \mathrm{d} \theta \mathrm{d} \phi$ over the unit sphere. The unit vectors can be expressed in terms of the spherical coordinates $\theta \in[0, \pi)$ and $\phi \in[0,2 \pi)$ as $\mathbf{r}=\sin (\theta) \cos (\phi) \mathbf{e}_{x}+\sin (\theta) \sin (\phi) \mathbf{e}_{y}+\cos (\phi) \mathbf{e}_{z}$ with $\left\{\mathbf{e}_{x}, \mathbf{e}_{y}, \mathbf{e}_{z}\right\}$ the reference Cartesian system. The integration over the unit sphere was performed using a Lebedev quadrature rule (Lebedev \& Laikov, 1999) for 77 st algebraic order of accuracy. This order, is determined by the concentration parameter $b$ and the form of the rupture region.

As commented above, the anisotropic part of the SEF is related to the collagen fibers present in the material. In a general situation with $\mathrm{N}$ families of fibers, the anisotropic part of the SEF can be expressed as

$\Psi_{\mathrm{ani}}=\sum_{j=1}^{N} \Psi_{\mathrm{f}}^{j}=\sum_{j=1}^{N}\left[\frac{1}{4 \pi} \int_{\mathbb{U}^{2}} n \rho_{\mathrm{f}} \psi_{\mathrm{f}} d A\right]_{\mathrm{j}}$

where $\Psi_{\mathrm{f}}^{j}$ is the strain energy density function for the $j$-nth fiber family, $n$ the chain density, $\rho_{\mathrm{f}}$ a statistical value associated with the fibrils dispersion and $\psi_{\mathrm{f}}$ the free energy function of the fibril. Since an analytical integration of (11) is not possible in general, a discretization of this equation as that presented in (10) is used

$\Psi_{\mathrm{ani}} \approx \sum_{j=1}^{N}\left[\sum_{i=1}^{m} n \rho_{i} w^{i} \psi\left(\bar{\lambda}^{i}\right)\right]$

where $\bar{\lambda}^{i}$ and $\psi\left(\bar{\lambda}^{i}\right)$ are the stretch ratio and the free energy function associated to each integration direction.

Regarding, the statistical distributions of the fibrils around a preferential orientation $\left(\rho_{\mathrm{f}}\right)$ in Eq. (10), a $\pi$ periodic von Mises orientation density function (ODF) (13) has been adopted in this work following (Alastrué et al., 2009)

$\rho(\theta)=4 \sqrt{\frac{b}{2 \pi}} \frac{\exp (b[\cos (2 \theta)+1])}{\operatorname{erfi}(\sqrt{2 b})}$,

where the concentration parameter $b \in \mathbb{R}^{+}$is a measure of the anisotropy; $b \rightarrow 0$ represents an isotropic material, and $b \rightarrow \infty$ a transversally isotropic one, and $\operatorname{Erfi}(x)$ is the imaginary error function.

Following the results of Eppell et al. (Eppell et al., 2006) which demonstrated a non-linear response of collagen fibrils, the contribution of each single collagen fibril in the micro scale is here defined by an exponential-type function as previously proposed by (Alastrué et al., 2009; Sáez et al., 2012)

$n \psi_{j}^{i}\left(\bar{\lambda}^{i}\right)=\left\{\begin{array}{ll}0, & \text { if } \bar{\lambda}^{i}<1 \\ \frac{c_{1}}{2 c_{2}}\left[\exp \left(c_{2}\left[\left[\bar{\lambda}^{i}\right]^{2}-1\right]^{2}\right)\right] & \text { if } \bar{\lambda}^{i} \geq 1\end{array}\right.$,

where $c_{1}>0$ is a stress-like parameters and $c_{2}>0$ is dimensionless.

\subsubsection{Microstructural damage model of vascular tissue}

Following Sáez et al. (Sáez et al., 2012), we postulate that the anisotropic part of the SEF defined in Eq. (1) is defined as

$\Psi_{\text {ani }} \approx \sum_{j=1}^{N}\left[\sum_{i=1}^{m} n \rho_{i} w^{i}\left[1-D_{i}\right] \psi_{j, 0}^{i}\left(\bar{\lambda}^{i}\right)\right]$

with $D_{i} \in[0,1]$ being the damage internal variables (Simo, 1987) for each integration direction respectively and $\psi_{0}\left(\bar{\lambda}^{i}\right)$ the effective strain energy density functions of the fibers defined in Eq. (14).

The damage variable $D_{i}$ is given by the damage energy release rate $\Xi_{i}=\sqrt{2 \psi_{0}(\bar{\lambda}(s))}$ where $\bar{\lambda}(s)$ the stretch ratio at pseudo-time $s \in \mathbb{R}$ in similar way to the phenomenological model (Eq. (7)) with

$\Xi_{i}^{*}=\max _{s \epsilon(-\infty, t]}\left(\sqrt{2 \psi_{0}(\lambda \overline{(s)})}\right)$

the maximum thermodynamic force achieved along the whole load history. Then, the damage criterion in the strain space is given by the condition

$\Phi_{i}\left(\bar{\lambda}(s), \Xi_{s, i}^{*}\right)=\sqrt{2 \psi_{0}(\bar{\lambda}(s))}-\Xi_{i}^{*} \leq 0$

Finally, the expression of the damage function is a sigmoidal type function as

$D_{i}=\frac{1}{1+\exp \left(-\alpha_{i}\left[\Xi_{i}^{*}-\gamma_{i}\right]\right)}$

where the parameters $\alpha_{i}$ and $\gamma_{i}$ defines the sigmoidal function in a similar way as presented in Eq (18) (Peña, 2011b). 


\subsection{Data fitting}

We fit the mechanical behavior of the tissue using the last loading curve till rupture for each sample (whole, intima, media and adventitia). It was necessary to perform two estimation processes. For the phenomenological and microstructural models, the elastic constitutive parameters $\left(\mu, k_{1}, k_{2}, \kappa, \theta\right)$ and $\left(\mu, c_{1}, c_{2}, b, \theta\right)$, respectively, were determined from the elastic region of the curves while the damage parameters $\left(\alpha_{m}, \alpha_{f}, \gamma_{m}, \gamma_{f}\right)$ and $\left(\alpha_{m}, \alpha_{f}, \gamma_{i}, \gamma_{i}\right)$, respectively, were fitted from the softening region of the curves. A total of 9 constitutive parameters should be fitted per sample for the phenomenological and microstructural models.

The tissue was assumed as incompressible (Carew et al., 1968), i.e. $\operatorname{det}(\mathbf{F})=\lambda_{1} \lambda_{2} \lambda_{3}=1$, where $\lambda_{i}$, $i=1,2,3$, the stretches in the principal directions. Considering a uniaxial tension test in the longitudinal or circumferential directions, then the Cauchy stress tensor become $\boldsymbol{\sigma}=\left[\sigma_{i i}, 0,0\right]$.

The fitting of the experimental damage data was performed using a Levenberg-Marquardt type minimization algorithm Marquardt (1963), by defining the objective function represented in Eq. (19).

$\chi^{2}=\sum_{i=1}^{n}\left[\left[\sigma_{\theta \theta}-\sigma_{\theta \theta}^{\Psi}\right]_{i}^{2}+\left[\sigma_{z z}-\sigma_{z z}^{\Psi}\right]_{i}^{2}\right]$,

In this expression, $\sigma_{\theta \theta}$ and $\sigma_{z z}$ are the Cauchy stress data obtained from the tests, $\sigma_{\theta \theta}^{\tilde{\Psi}}=\lambda_{\theta} \frac{\partial \Psi}{\partial \lambda_{\theta}}$ and $\sigma_{z z}^{\tilde{\Psi}}=$ $\lambda_{z} \frac{\partial \Psi}{\partial \lambda_{z}}$ are the Cauchy stresses for the $i$ th point computed using the SEF defined in Eq. (3), and $n$ is the number of data points. The coefficient of determination $R^{2} \in[0,1]$ was computed for each fitting where $R^{2} \geq 0.85$ typically represents a good fit to the experimental data.

\section{Results}

A total of 11 strips were discarded during postprocessing due to the unsuccessful layer separation (intima, media, or adventitia), damage occurred close to the grips or poor speckle patterns, 5 strips corresponding to intima samples of IAA, or occurring close to the clamps ( 4 for DTA and 2 for IAA). In all cases, a new sample was taken in order to have a minimum of four circumferential and longitudinal samples per animal (whole, intima, media and adventitia strips). Figure 1 shows photographs of axial strips from the adventitia, media and intima after 30 min of equilibration from a DTA sample and the media sample at the beginning of the test, before rupture and after rupture where the damage occurred close to the central part of the strip as a valid test.

Thickness of the total wall for the DTA and IAA is as follows: $2.51 \pm 0.60[\mathrm{~mm}]$ and $1.48 \pm 0.33[\mathrm{~mm}]$, respectively; and thickness for DTA and IAA is $0.27 \pm 0.09$ [mm] and $0.24 \pm 0.08[\mathrm{~mm}]$ for intima, $1.51 \pm 0.17[\mathrm{~mm}]$ and $0.69 \pm 0.29[\mathrm{~mm}]$ for media, and $0.74 \pm 0.16[\mathrm{~mm}]$ and $0.46 \pm 0.07[\mathrm{~mm}]$ for adventitia.

\subsection{Mechanical tests}

A representative uniaxial mechanical response (Cauchy stress vs. stretch) up to fracture for the complete and for separated layer samples are plotted in Figure 2 for descending thoracic and abdominal sample specimens $\mathrm{V}$ and VI, respectively. The different behavior between circumferential and longitudinal directions and between descending thoracic and abdominal aorta are clearly depicted for all layers (intima, media and adventitia).

Overall, a large variation in the results are presented in Figures 3 and 4 for descending thoracic and abdominal aorta but marked mechanical heterogeneity was found. The variation in the curve profiles may be related to structural variations between individual specimens. The dispersion of the stress-stretch curves is much higher for the intima and adventitia than for the media samples and is much smaller for samples tested in the circumferential than in the longitudinal direction. All tissue samples exhibit a pronounced nonlinear mechanical response, and the anisotropic behavior for elastic and rupture response is more marked in the abdominal aorta. The complete and media circumferential samples displayed higher fracture stress and the axial specimens higher fracture stretches for both thoracic and abdominal aorta, but these differences are more rmarked for abdominal samples. In addition, the abdominal samples are stiffer than the descending thoracic samples, the most important differences being between the descending thoracic and abdominal behaviors in the circumferential direction.

The complete samples had a fragmented response under loading (flattening and dips) in both the circumferential and longitudinal directions associated with localized failure of the arterial layers. The intima and adventitia of the descending aorta showed an isotropic response while the complete and media samples had similar anisotropic responses, showing that the mechanical response of the descending thoracic artery is dominated by the media behavior. The abdominal samples showed a greater increase in fracture stress and stretch in both directions than the descending thoracic aorta samples. In the longitudinal direction, 


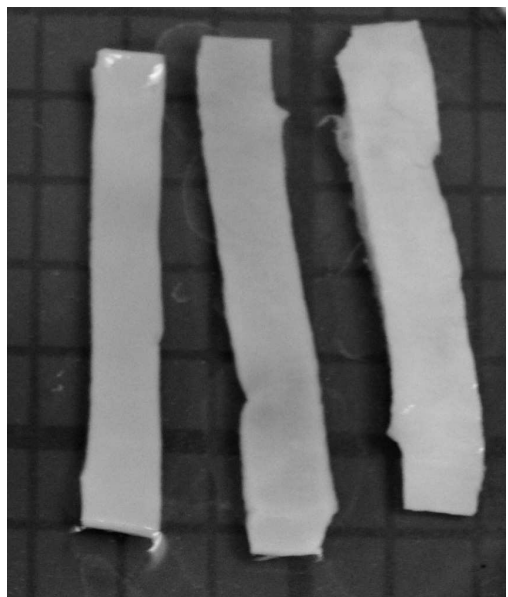

(a) Separated layer strips

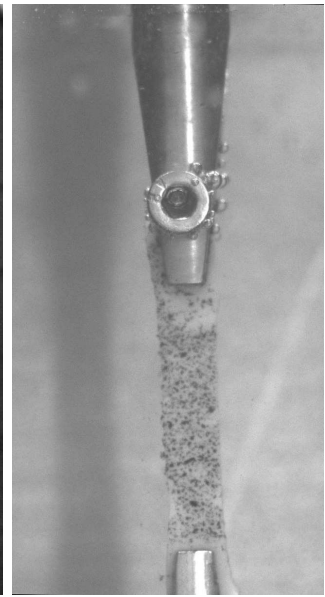

(b) Before test

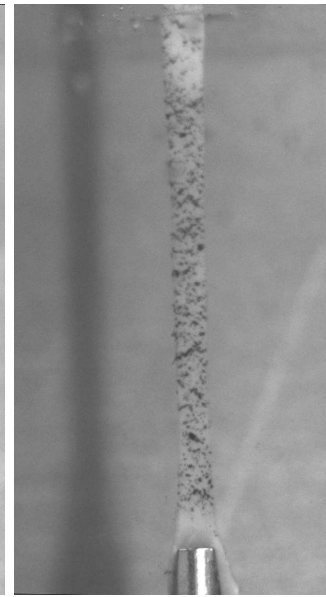

(c) Before rupture

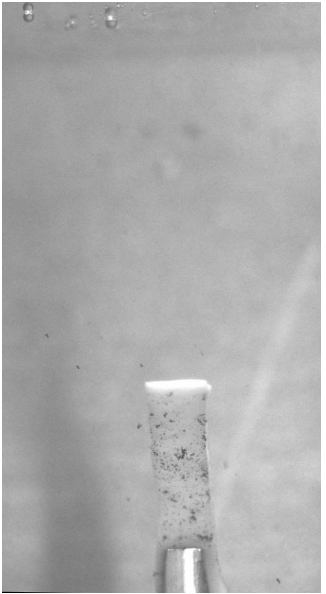

(d) After rupture

Fig. 1 Photographs of (a) axial strips from the adventitia, media and intima after 30 min of equilibration from the DTA, (b) media axial sample at the beginning of the test, (c) before rupture and (d) after rupture.

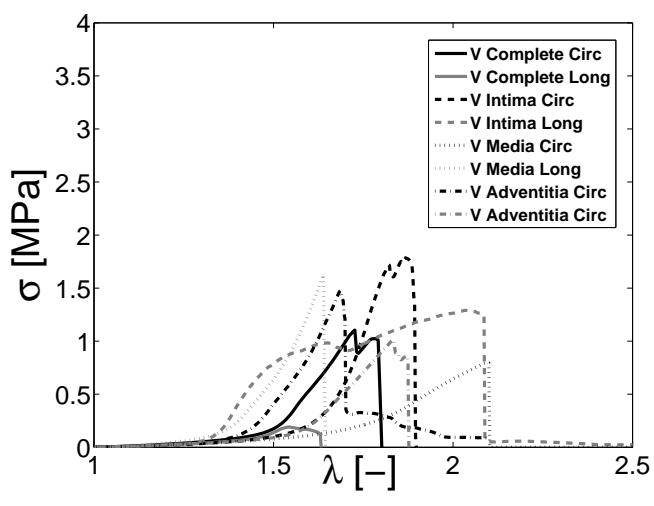

(a) Sample V DTA

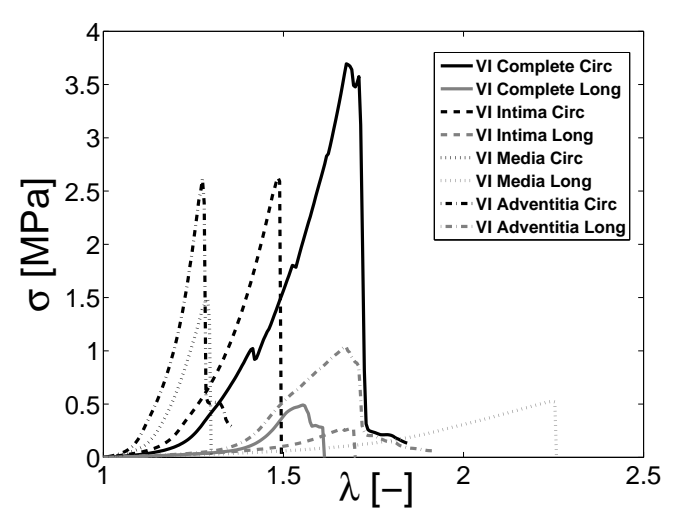

(b) Sample VI IAA

Fig. 2 Representative Cauchy stress vs. stretch behavior for the complete and for separated layer samples of DTA (V) and IAA (VI) specimens. the fracture stress and stretch of the intima are similar to those of the control samples..

Figures 5.a and 5.b show column plots of the rupture $\operatorname{stretch}\left(\lambda_{r}[-]\right)$ and rupture stress $\left(\sigma_{r}[\mathrm{MPa}]\right)$ for DTA and IAA corresponding to the circumferential and longitudinal directions. Table 1 also shows the Mean and Standard Deviation (SD) of the maximum rupture values of stress [MPa] and stretch [-] for both circumferential and longitudinal samples. In general, $\lambda_{r}$ values for the longitudinal direction are higher than the circumferential for both, DTA and IAA, locations, especially for the media layer. Also, $\lambda_{r}$ values for the DTA samples are higher than those of the IAA samples for both directions except for Intima and adventitia layer of the IAA samples. In contrast, $\sigma_{r}$ was higher for the IAA locations than the DTA and this ultimate stress was lower in the circumferential direction than in the longitudinal, except for the intima layer which showed lower $\sigma_{r}$ for IAA positions and the longitudinal direction.

Here, we also analyze possible statistically-relevant differences $(p<0.05)$ between the rupture values of the DTA and IAA tissue layers. There were significant differences in the $\lambda_{r}$ between the circumferential and longitudinal directions in media layer for the DTA and the media and adventitia for the IAA samples $(p<0.05)$. In addition, there were significant differences in $\lambda_{r}$ between the whole samples and media samples and between the adventitia and media layers only for longitudinal directions of IAA; and between the media and intima layers for the longitudinal directions of the DTA samples. As regards sample loca- 


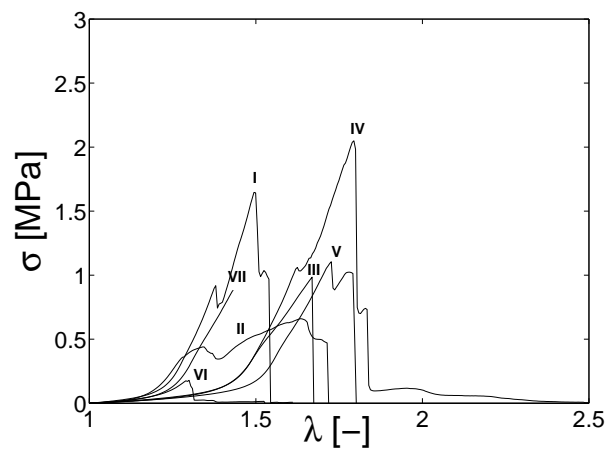

(a) Complete DTA Circumferential

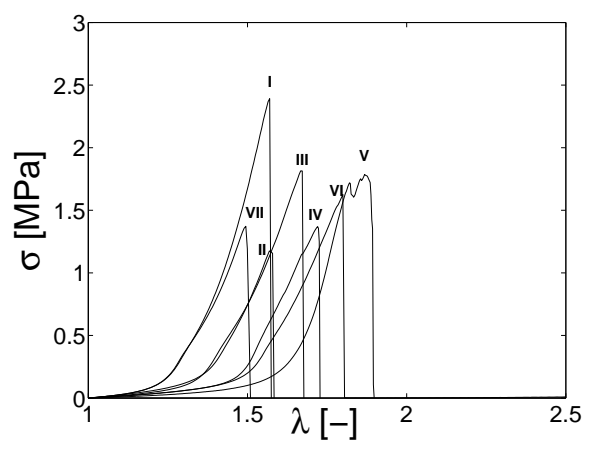

(c) Intima DTA Circumferential

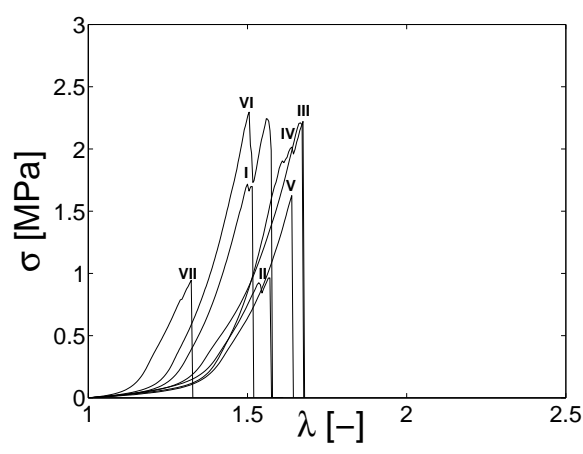

(e) Media DTA Circumferential

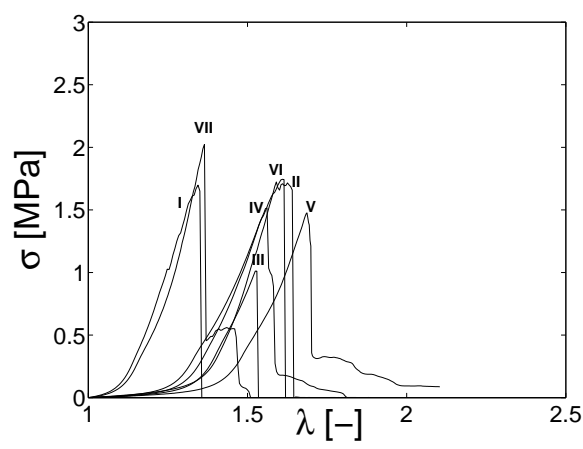

(g) Adventitia DTA Circumferential

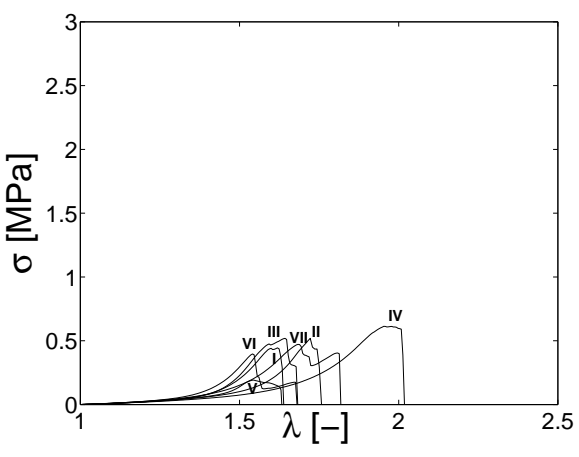

(b) Complete DTA Longitudinal

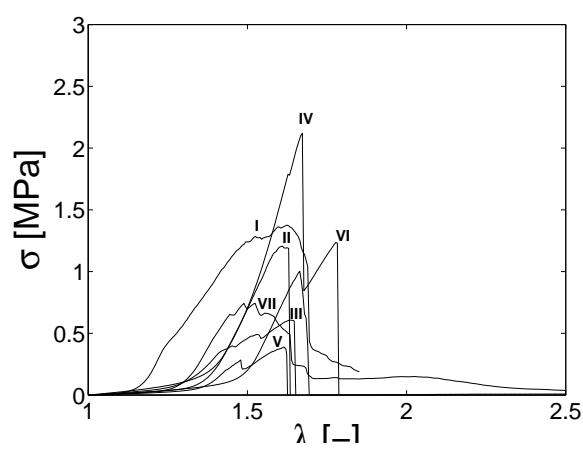

(d) Intima DTA Longitudinal

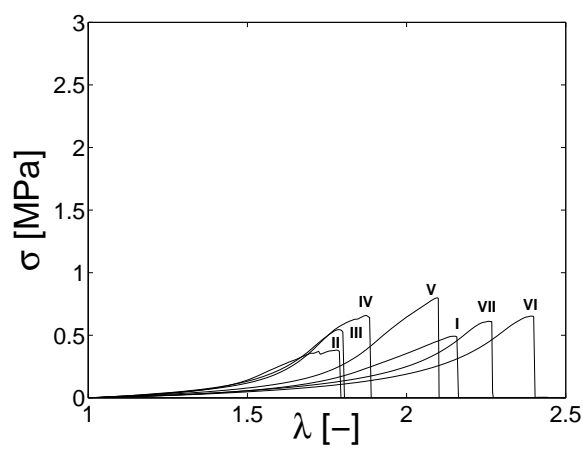

(f) Media DTA Longitudinal

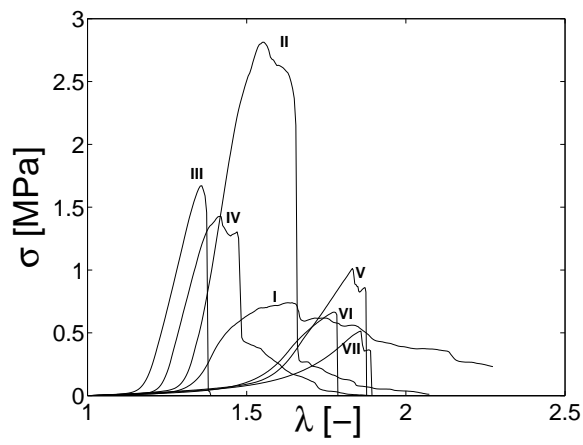

(h) Adventitia DTA Longitudinal

Fig. 3 Uniaxial tensile stress-stretch curves for separate layer samples I-VII of DTA. 


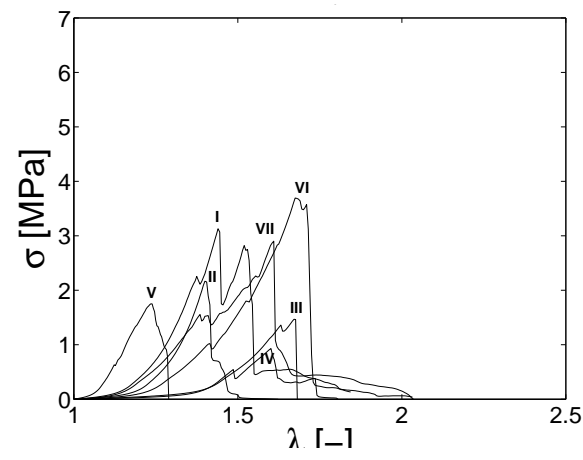

(a) Complete IAA Circumferential

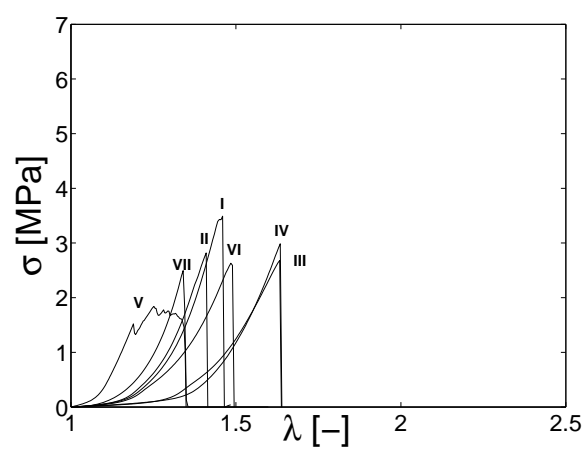

(c) Intima IAA Circumferential

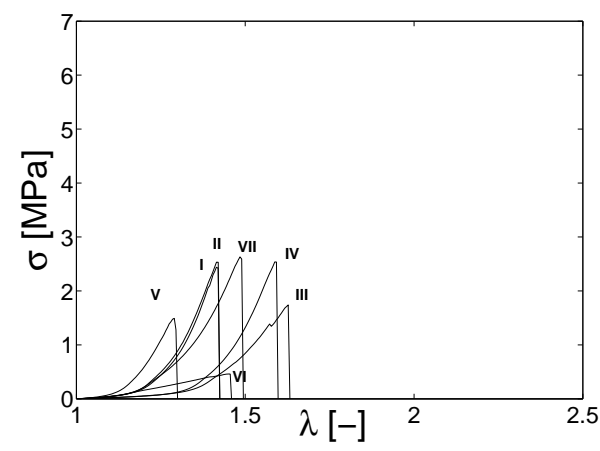

(e) Media IAA Circumferential

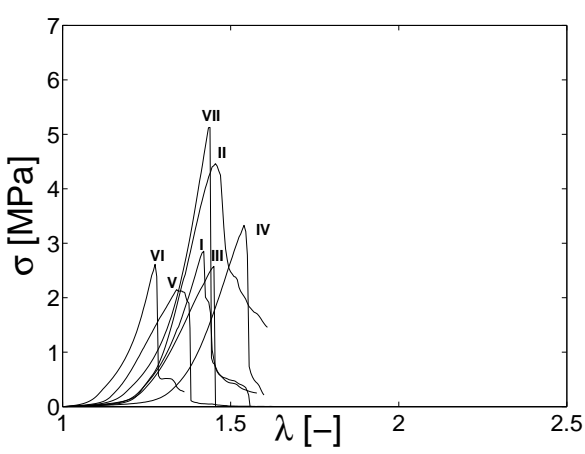

(g) Adventitia IAA Circumferential

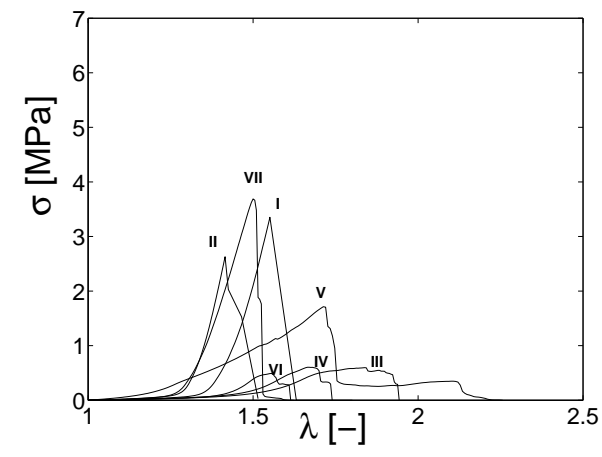

(b) Complete IAA Longitudinal

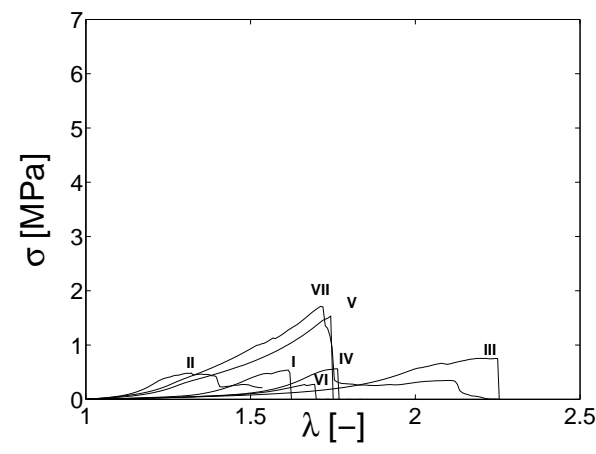

(d) Intima IAA Longitudinal

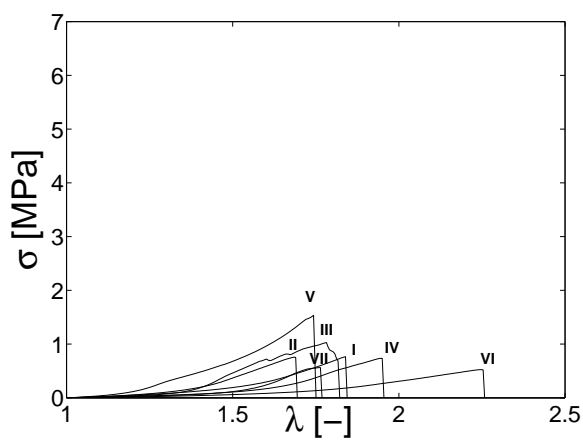

(f) Media IAA Longitudinal

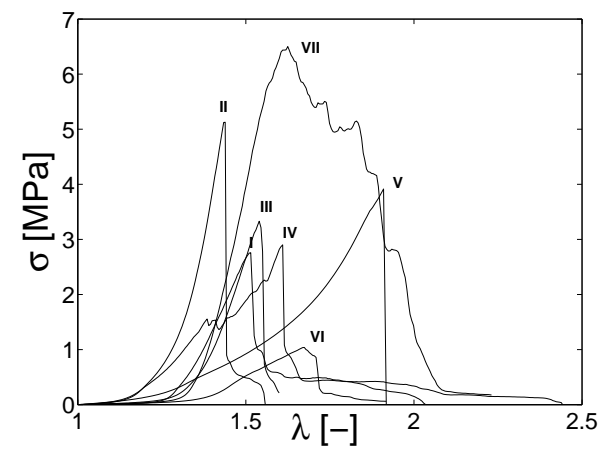

(h) Adventitia IAA Longitudinal

Fig. 4 Uniaxial tensile stress-stretch curves for separate layer samples I-VII of IAA. 


\begin{tabular}{|c|c|c|c|c|c|c|c|c|}
\hline & \multicolumn{4}{|c|}{ DTA } & \multicolumn{4}{|c|}{ IAA } \\
\hline & "Whole & Intima & Media & Adventitia & "Whole & Intima & Media & $\overline{\text { Adventitia }}$ \\
\hline$\overline{\lambda_{r} \operatorname{circ}}$ & $1.58 \pm 0.17$ & $1.65 \pm 0.16$ & $1.54 \pm 0.12$ & $1.51 \pm 0.13$ & $1.51 \pm 0.16$ & $1.45 \pm 0.14$ & $1.43 \pm 0.13$ & $1.41 \pm 0.08$ \\
\hline$\lambda_{r}$ long & $1.61 \pm 0.13$ & $1.61 \pm 0.06$ & $1.97 \pm 0,23$ & $1.61 \pm 0.20$ & $1.60 \pm 0.14$ & $1.67 \pm 0.27$ & $1.83 \pm 0.19$ & $1.67 \pm 0.23$ \\
\hline$\sigma_{r} \operatorname{circ}$ & $0.83 \pm 0.57$ & $1.42 \pm 0.32$ & $1.70 \pm 0.64$ & $1.43 \pm 0.40$ & $2.091 \pm 0.99$ & $2.60 \pm 0.54$ & $1.62 \pm 0.75$ & $3.36 \pm 1.15$ \\
\hline$\sigma_{r}$ long & $0.45 \pm 0.16$ & $1.06 \pm 0.53$ & $0.55 \pm 0.14$ & $1.34 \pm 1.09$ & $1.38 \pm 1.36$ & $0.70 \pm 0.57$ & $0.83 \pm 0.41$ & $3.47 \pm 2.59$ \\
\hline
\end{tabular}

Table 1 Mean \pm SD of rupture values of stress $[\mathrm{MPa}]$ and stretch[-], $\left(\sigma_{r}\right.$ and $\left.\lambda_{r}\right)$ respectively, for both circumferential and longitudinal samples

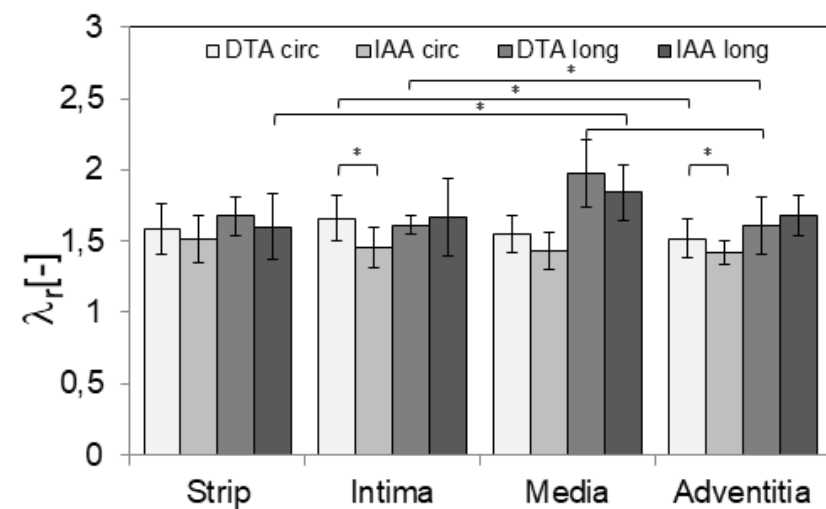

(a) Rupture stretch

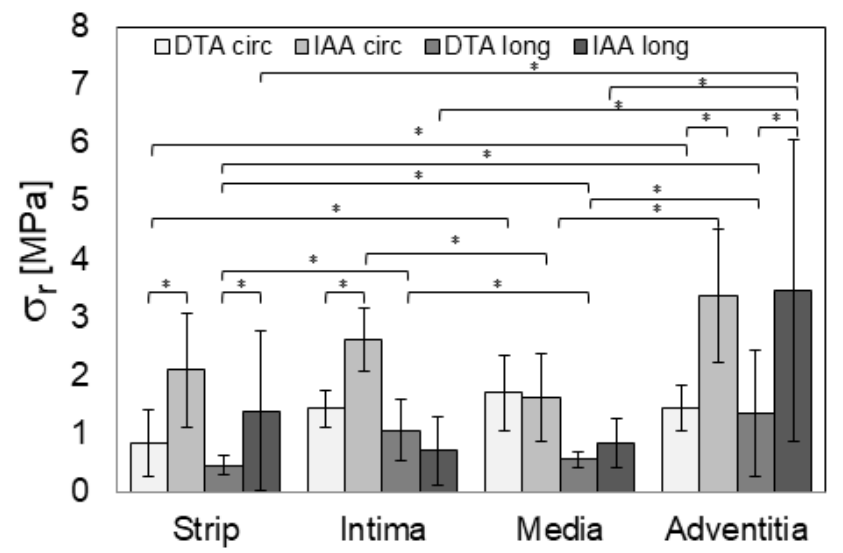

(b) Rupture stress

Fig. 5 Maximum rupture values of stretch [-] and stress [MPa], $\left(\sigma_{r}\right.$ and $\lambda_{r}$ ) respectively, for both circumferential and longitudinal samples. For the comparisons between $\sigma_{r}$ and $\lambda_{r}$ for DTA and IAA corresponding to the circumferential and longitudinal directions, the means which are significantly different are marked with $* p<0.05$.

tions, there were significant differences in $\lambda_{r}$ between the DTA and IAA samples for the intima and adventitia in the the circumferential direction, but not for longitudinal $(p=0.42)$.

In the case of $\sigma_{r}, p<0.05$ for the whole artery and media layer of the DTA samples and intima and media layers of the IAA samples confirms that there is sta- tistical significance in $\sigma_{r}$ between the circumferential and longitudinal directions. Also, there are statistically significant differences $(p<0.05)$ in $\sigma_{r}$ between the whole artery and adventitia samples for the longitudinal and circumferential directions for the DTA and IAA samples. There was significant differences $(p<0.05)$ between the whole and intima DTA samples for the longitudinal direction only, and between the adventitia and media for IAA samples and between the intima and media for DTA samples also in the longitudinal direction only. Finally, the differences in $\sigma_{r}$ between the whole and media samples were statistically $(p<0.05)$ significant for the DTA samples in both directions.

\subsection{Histological analysis}

Histological sections of the samples stained with Hematoxiline-Eosine are shown in Figure 6. Correct anatomical separation of the arterial tissue into its three corresponding layers were checked. For most specimens only a few laminae of the media were still attached to the intima or adventitia. The threshold of 6 lamellas to exclude specimens with an incorrect layer separation were considered.

\subsection{Data fitting}

\subsubsection{Phenomenological anisotropic hyperelastic damage model}

The stress-stretch plots in Figures 7 and 8 show the mechanical response and fitting results for the whole artery and each separated layer, of a representative DTA sample (III) and IAA sample (IV), using the phenomenological damage model. The correlation between the experimental stress-stretch data and the response of the proposed constitutive model was satisfactory. The worst fit is always when the damage response is highly anisotropic and the maximum loads for the circumferential an and longitudinal directions are totally different. For example, for the media layer of the DTA sample III, the maximum loads before rupture in the circumferential and longitudinal direction 


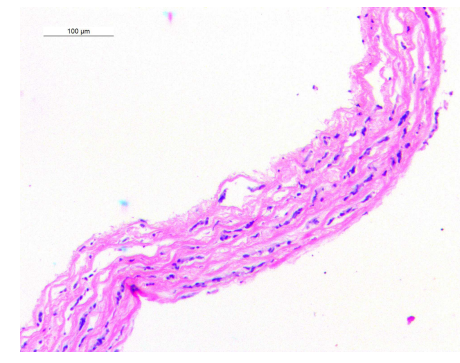

(a) Intima DTA

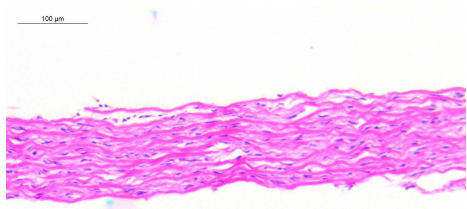

(d) Intima IAA

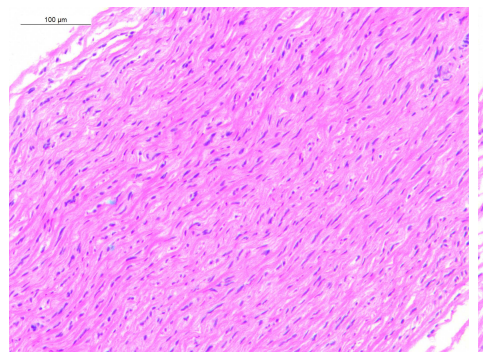

(b) Media DTA

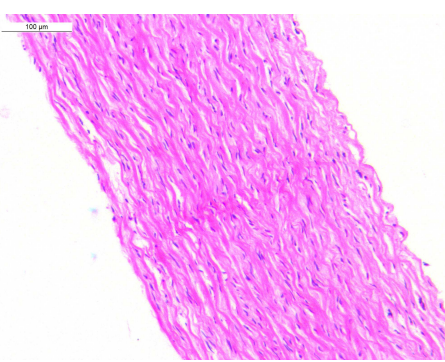

(e) Media IAA

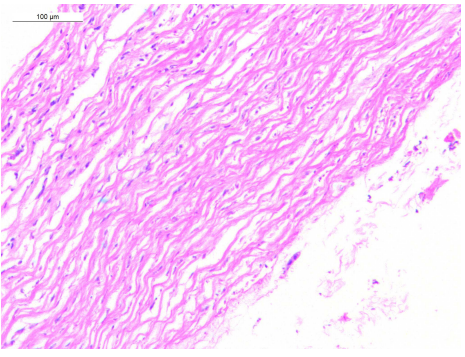

(c) Adventitia DTA

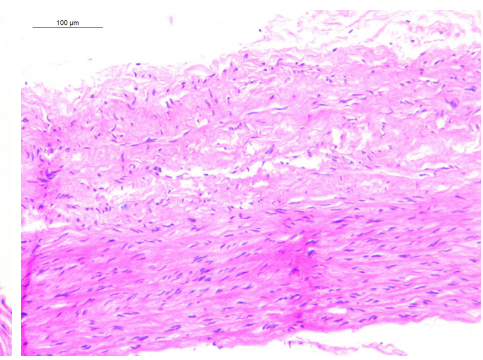

(f) Adventitia IAA

Fig. 6 Hematoxylin-and-eosin-stained sections $5 \mu \mathrm{m}$ from wall layers after anatomic separation.

are 2.35 and 0.6 $\mathrm{MPa}$, respectively. In this case, the fitting curve for the longitudinal direction underestimates the stress due to the coupled character of the damage model. A similar response is obtained for the intima layer of the IAA sample IV. Also, samples with a narrow damage zone (of the stress-stretch curve), which indicates rapid damage and rupture have a better fit than samples suffering a mild damage process identifiable by the smooth round-shaped damage zone, see Figures 7.b and d and 8.c.

The experimental data curves were fitted using the proposed phenomenological model following the procedure explained in Section 3.3. The results of the fitting to the phenomenological model for the DTA and IAA samples are shown in Tables 2 and 3, respectively. In most cases the $R^{2}$ is higher than 0.8. In general, the parameter $\kappa$ ranges between 0.15 and 0.33 for the DTA samples showing a higher fiber dispersion, and ranges between 0.12 and 0.20 for the IAA samples which reveals higher directionality of the collagen fibers. The phenomenologically identified parameter $\theta$ (the angle of the collagen fibers) is always higher than $45^{\circ}$ for the IAA samples and almost always higher than $45^{\circ}$ for the DTA samples demonstrating a higher stiffness in the circumferential direction. Regarding the material parameters, the parameters $\gamma_{f}$ that defines the value $\Xi_{f_{t}}$ such that $D_{f}\left(\Xi_{f_{t}}\right)=0.5$ ranges between
0.27 and $0.46[\mathrm{MPa}]$ for the DTA and IAA samples, except for the media of the IAA specimens, showing similar fiber damage accumulation independent of the position and geometrical fiber parameters $(\kappa$ and $\theta)$. However, the material parameter $\alpha_{f}$ that controls the slope of the damage function (brittle response) ranges in values from 39 to 206, showing strong dependency on the positions and microstructure of the tissue (dispersion and orientation of the collagen fibers). The matrix damage parameters $\left(\alpha_{m}\right.$ and $\left.\gamma_{m}\right)$ also have different values depending on the position and layer.

\subsubsection{Microstructural damage model}

The stress-stretch plots in Figures 9 and 10 show the mechanical response and fitting results for the whole artery and each separated layer, of a representative DTA sample (III) and IAA sample (IV) using the microstructural damage model. The correlation between the experimental stress-stretch data and the response of the proposed constitutive model was less satisfactory and worse than the fitting using the phenomenological damage model.

The worst fit is always when the elastic response is quasi-isotropic and the damage response is highly anisotropic. The maximum loads for the circumferential and longitudinal directions are totally different, as 


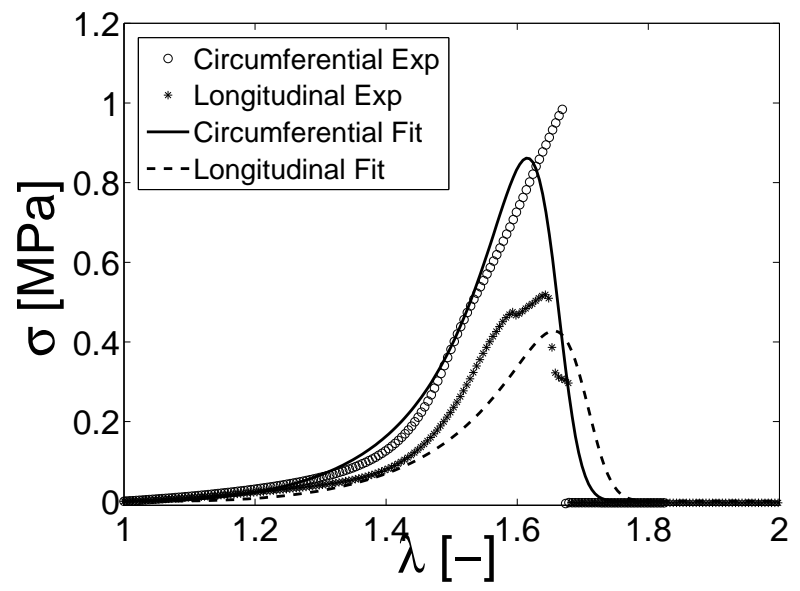

(a) Whole artery

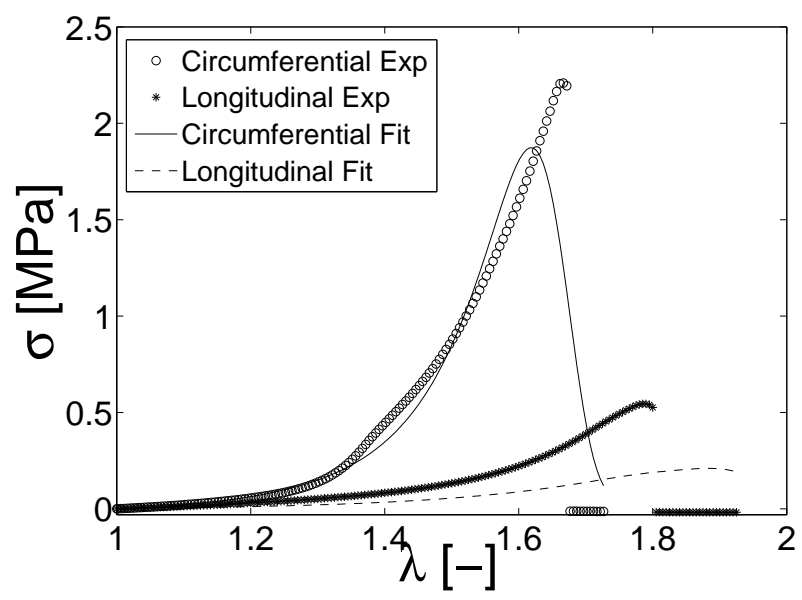

(c) Media

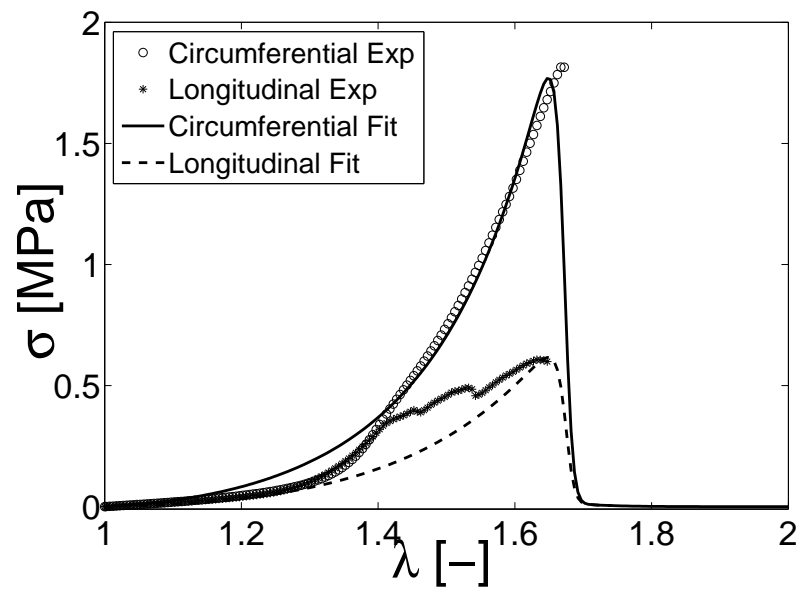

(b) Intima

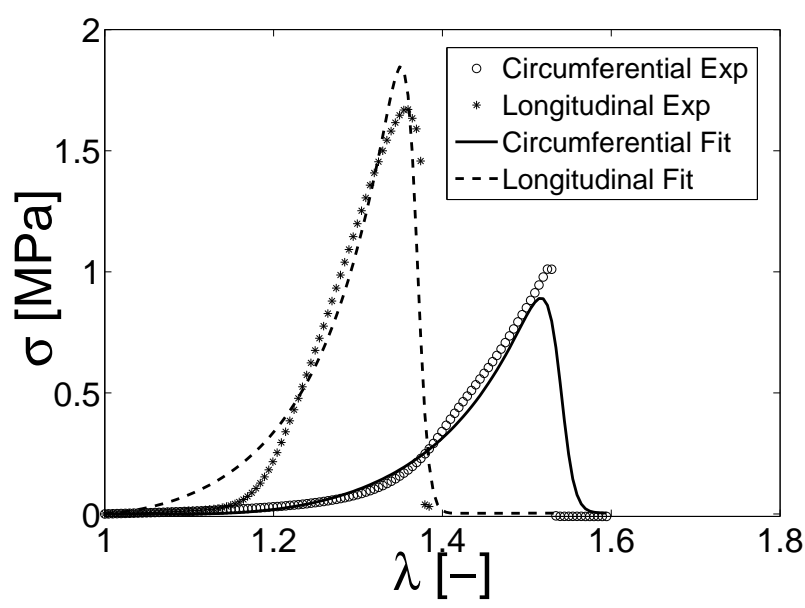

(d) Adventitia

Fig. 7 Representative DTA curves (III) and simulation results for (a) whole artery, (b) intima, (c) media and (d) adventitia with the phenomenological damage model.

occurs with the phenomenological model. For example, see Figures 9.b and d and 10.a for the intima layer of DTA sample III or the whole artery of IAA sample IV. In addition, when the longitudinal direction is softer than the circumferential for the elastic part, the damage response is isotropic and the maximum loads for the circumferential and longitudinal directions are very similar, the stress in the longitudinal direction is underestimated due to the coupled character of the damage model. For example, see Figure 9.d. for the adventitia layer of DTA sample III In general, samples with a narrow damage zone, which indicates a rapid damage and rupture, have a worse fit than samples which have suffered a mild damage process identifiable by the smooth round-shaped damage zone. Due to the discrete local integration character of the microstructural damage model, when one fiber associated to one direction of integration fails, other fibrils associated to the other integration direction support the load, causing a non-smother stress response.

The experimental data curves were also fitted using the proposed microstructural damage model following the procedure explained in Section 3.3. The results of the fitting are shown in Tables 4 and 5. In most cases the $R^{2}$ is lower than the corresponding value for the phenomenological model in some cases with $R^{2}<$ 0.4. This demonstrates that from the fitting point of view, the phenomenological damage model is better than the microstructural model. The worst fit was for the intima layer of the DTA samples with a value of 


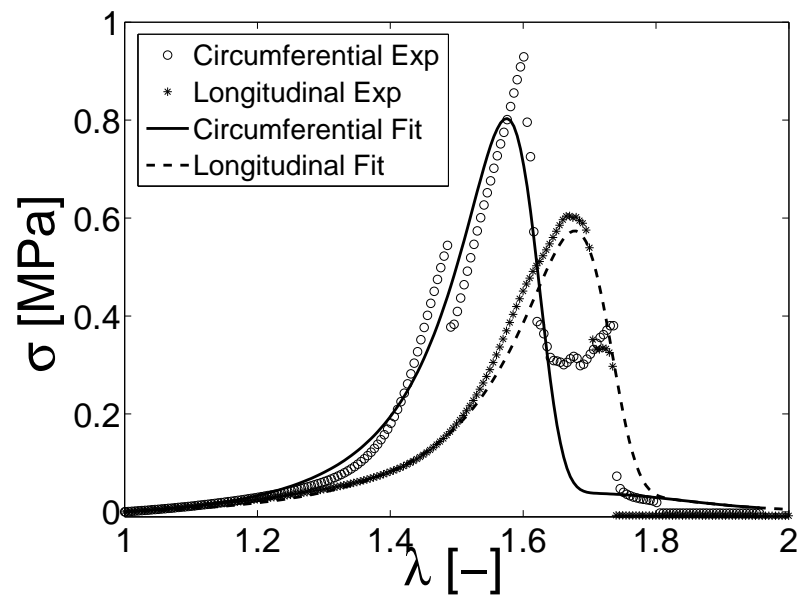

(a) Whole artery

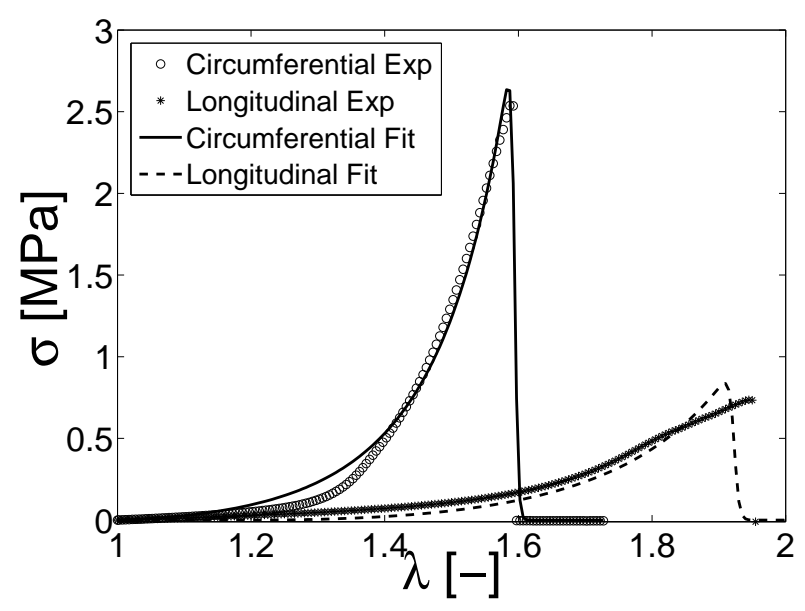

(c) Media

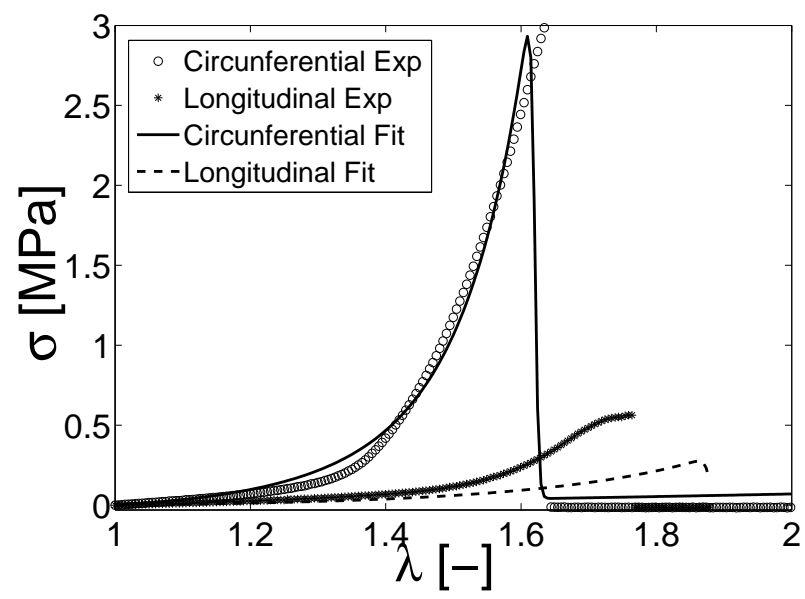

(b) Intima

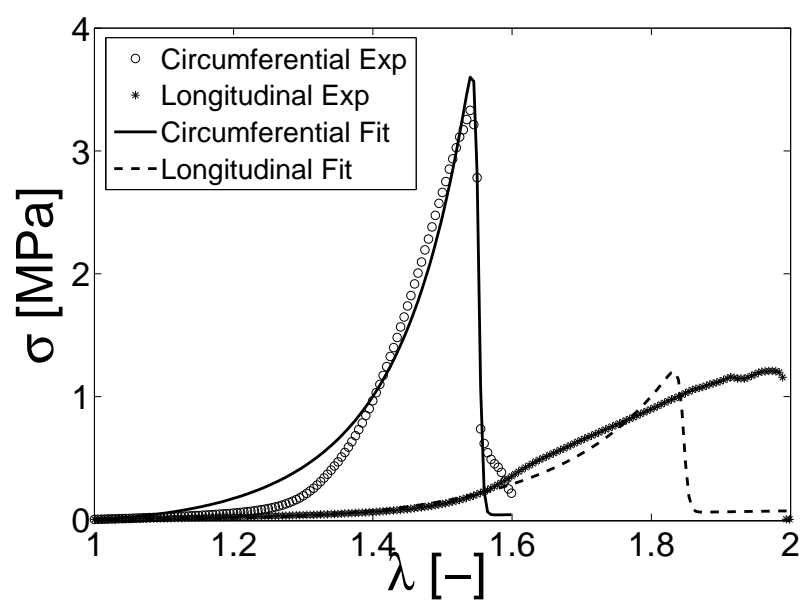

(d) Adventitia

Fig. 8 Representative IAA curves (IV) and simulation results for (a) whole artery, (b) intima, (c) media and (d) adventitia with the phenomenological damage model.

$R^{2}=0.47 \pm 0.16$ due to the isotropic character of the elastic part and the anisotropic character of the damaged part. The best fitting was for the media layer of the DTA with a value of $R^{2}=0.63 \pm 0.20$. Finally, note that the fiber damage parameters $\left(\alpha_{f}\right.$ and $\left.\gamma_{f}\right)$ are very similar for all layers and samples due to the fact that fiber rupture properties.

\section{Discussion}

Aortic functionality can be impaired by several cardiovascular diseases that cause damage or tearing such as aneurysm or dissection leading to a high risk of mortality. Most studies on softening, damage and failure of arterial wall properties have char- acterized arteries as single layer homogeneous structures (García et al., 2013; Duprey et al., 2016; Maher et al., 2012). However, arteries are heterogeneous three-layered structure (intima, media, and adventitia) with layer-specific mechanical properties. Thus a better understanding of their mechanical function at the tissue level requires layer-specific data. Few studies consider the layer specific damage properties of the aorta. Weisbecker et al. (2012) addresses the layer specific softening response of the human aorta. However, their study only presented the softening response of the layers and there was no data about the failure properties. Kim et al. (2012) performed a bulge inflation tests on media and adventitia layers of human aneurismal aortic tissues up to failure. However, 


\begin{tabular}{|c|c|c|c|c|c|c|c|c|c|c|}
\hline Specimen & $\mu$ & $k_{1}$ & $k_{2}$ & $\theta$ & $\kappa$ & $\alpha_{m}$ & $\gamma_{m}$ & $\alpha_{f}$ & $\gamma_{f}$ & $R^{2}$ \\
\hline \multicolumn{11}{|c|}{ Whole artery } \\
\hline$I$ & 0.011 & 1.1455 & 18.2856 & 1.81 & 0.3157 & 99.9080 & 0.2244 & 7.0229 & 0.1012 & 0.8480 \\
\hline$I I$ & 0.0105 & 0.4818 & 0.0089 & 28.26 & 0.2163 & 115.1949 & 0.2364 & 92.5875 & 0.3622 & 0.4076 \\
\hline$I I I$ & 0.010 & 0.3688 & 10.1567 & 14.46 & 0.3149 & 12.0080 & 0.0010 & 18.4086 & 0.2448 & 0.8496 \\
\hline$I V$ & 0.0252 & 0.1107 & 1.3033 & 30.56 & 0.1382 & 10.0924 & 0.2684 & 10.6711 & 0.4859 & 0.8876 \\
\hline$V$ & 0.0162 & 0.1527 & 1.0621 & 24.91 & 0.2054 & 100.0000 & 0.1807 & 20.5776 & 0.6000 & 0.9181 \\
\hline$V I$ & 0.0151 & 0.5116 & 0.0156 & 3.64 & 0.3041 & 7.3545 & 2.9048 & 114.9979 & 0.1236 & 0.2583 \\
\hline$V I I$ & 0.0308 & 1.4091 & 26.4467 & 10.83 & 0.2833 & 192.5518 & 0.0030 & 10.8678 & 0.0100 & 0.9516 \\
\hline Mean & 0.01697 & 0.5971 & 8.1827 & 16.36 & 0.2539 & 76.7299 & 0.5455 & 39.3047 & 0.2753 & 0.7315 \\
\hline$S D$ & 0.0080 & 0.4944 & 10.5757 & 11.71 & 0.0684 & 70.0183 & 1.0460 & 44.7707 & 0.2167 & 0.2780 \\
\hline \multicolumn{11}{|l|}{ Intima } \\
\hline$I$ & 0.0013 & 5.343 & 24.0885 & 1.00 & 0.3237 & 86.7862 & 0.0010 & 0.3631 & 0.3170 & 0.9394 \\
\hline II & 0.004 & 0.2531 & 2.4286 & 34.67 & 0.1808 & 100.0000 & 0.2222 & 98.9707 & 0.3359 & 0.9392 \\
\hline III & 0.0100 & 0.3724 & 2.3497 & 39.44 & 0.1823 & 96.46 & 0.2071 & 9.2303 & 1.0986 & 0.9119 \\
\hline$I V$ & 0.0100 & 0.0292 & 81.7298 & 64.55 & 0.2958 & 42.1724 & 0.2794 & 54.6172 & 0.0897 & 0.7221 \\
\hline$V$ & 0.0085 & 0.3043 & 8.2914 & 56.27 & 0.2583 & 99.5847 & 0.2000 & 6.5233 & 0.4063 & 0.1478 \\
\hline$V I$ & 0.0100 & 0.1460 & 1.1101 & 40.25 & 0.1182 & 16.9875 & 0.1603 & 201.3337 & 0.3751 & 0.7247 \\
\hline$V I I$ & 0.0100 & 1.0165 & 94.5125 & 0.01 & 0.3222 & 3.6484 & 4.5073 & 8.5398 & 0.1900 & 0.5370 \\
\hline Mean & 0.0076 & 1.0663 & 30.6443 & 33.77 & 0.2401 & 63.6627 & 0.7967 & 54.2254 & 0.4018 & 0.7031 \\
\hline$S D$ & 0.0035 & 1.9121 & 40.2087 & 24.98 & 0.0804 & 41.7597 & 1.6384 & 74.0849 & 0.3266 & 0.2863 \\
\hline \multicolumn{11}{|l|}{ Media } \\
\hline$I$ & 0.0098 & 1.0737 & 16.8578 & 1.87 & 0.2662 & 87.49 & 0.2204 & 0.2926 & 0.1100 & 0.9184 \\
\hline$I I$ & 0.01586 & 0.3743 & 2.7960 & 27.64 & 0.2500 & 19.7945 & 0.1107 & 117.0280 & 0.3357 & 0.9374 \\
\hline$I I I$ & 0.0072 & 0.0921 & 2.7574 & 34.14 & 0.0583 & 92.6382 & 0.2776 & 4.6533 & 0.5000 & 0.8288 \\
\hline$I V$ & 0.0100 & 0.1784 & 1.0407 & 33.92 & 0.0805 & 191.8825 & 0.2762 & 198.1099 & 0.5409 & 0.9150 \\
\hline$V$ & 0.0060 & 0.2261 & 2.8796 & 20.86 & 0.2442 & 70.0336 & 0.2000 & 58.1134 & 0.3684 & 0.8443 \\
\hline$V I$ & 0.0080 & 0.3855 & 1.2380 & 0.39 & 0.1916 & 352.4423 & 0.6015 & 496.0084 & 0.0510 & 0.9164 \\
\hline$V I I$ & 0.0100 & 0.1808 & 3.1381 & 26.86 & 0.0 & 268.2386 & 0.7191 & 99.7525 & 0.1675 & 0.5768 \\
\hline Mean & 0.0095 & 0.3587 & 4.3868 & 20.82 & 0.1558 & 154.6456 & 0.3436 & 139.1368 & 0.2962 & 0.8481 \\
\hline$S D$ & 0.0031 & 0.3329 & 5.5625 & 14.19 & 0.1077 & 120.4936 & 0.2259 & 171.6602 & 0.1912 & 0.1265 \\
\hline \multicolumn{11}{|l|}{ Adventitia } \\
\hline$I$ & 0.0120 & 2.8078 & 5.2094 & 72.00 & 0.2784 & 100.00 & 0.2073 & 0.0027 & 0.5000 & 0.8615 \\
\hline II & 0.0078 & 0.9591 & 40.6955 & 63.76 & 0.3061 & 82.7346 & 0.0823 & 4.6479 & 0.2050 & 0.6148 \\
\hline$I I I$ & 0.0081 & 2.4371 & 20.3621 & 76.35 & 0.2892 & 4.6667 & 9.4180 & 45.6326 & 0.3295 & 0.9291 \\
\hline$I V$ & 0.0098 & 2.2316 & 42.9244 & 77.30 & 0.3125 & 1.9455 & 1.3191 & 13.2685 & 0.2461 & 0.9258 \\
\hline$V$ & 0.0100 & 0.1570 & 5.0353 & 38.00 & 0.2151 & 23.0410 & 7.5532 & 6.9720 & 0.7076 & 0.9159 \\
\hline$V I$ & 0.0083 & 0.6396 & 23.0510 & 0.00 & 0.2851 & 98.8049 & 0.1400 & 7.6807 & 0.0100 & 0.7901 \\
\hline$V I I$ & 0.0100 & 0.5021 & 2.0671 & 29.84 & 0.0454 & 410.8192 & 0.0020 & 498.9927 & 0.4247 & 0.8840 \\
\hline Mean & 0.0094 & 1.3906 & 19.9064 & 51.04 & 0.2474 & 103.1445 & 2.6745 & 82.4567 & 0.3461 & 0.8458 \\
\hline$S D$ & 0.0014 & 1.0702 & 16.9681 & 29.31 & 0.0945 & 142.2818 & 4.0308 & 184.2948 & 0.2249 & 0.1128 \\
\hline
\end{tabular}

Table 2 Material constants obtained for the descending thoracic aorta (DTA) curves for the phenomenological damage model. Constants $\mu$ and $k_{1}$ are in MPa, $\gamma_{m}, \gamma_{f}$ are in $\sqrt{M P a}, \theta$ in degrees, $k_{2}, \kappa, \alpha_{m}, \alpha_{f}$ and $R^{2}$ are dimensionless.

they did not performed inflation test of intima layer or healthy tissue.

In this paper, we characterize the failure properties of the intact wall and each separated layer (intima, media and adventitia) of the descending thoracic and infrarenal abdominal aorta. We hypothesize that the layer-separated failure properties of aorta may be dependent on arterial location in the arterial tree and we report values of constitutive parameters for phenomenological and microstructural damage models. The incorporation of damage in mechanical constitutive models of arteries is a prerequisite for a better understanding of clinical treatments involving supraphysiological loading. To the best of the authors' knowledge, this study is the first to report direct measurements of the layer-specific failure mechanical responses of arteries and the determination of damage constitutive parameters using damage models.

Progress in experimental mechanics has significantly contributed to characterizing the mechanics of arteries (Alastrué et al., 2008; Peña et al., 2009; Peña \&
Doblare, 2009; Weisbecker et al., 2012; García et al., 2013). Analyzing these experiments, four important softening effects associated with arteries may be distinguished (Peña, 2014). First, there is the dependence of the mechanical response on the previously attained maximum load level very similar to the well-known Mullins effect in rubber-like materials (Mullins, 1947; Diani et al., 2009; Weisbecker et al., 2012). Another typical phenomenon known as preconditioning or hysteresis is characterized by continuous softening at the same load level after the first loading until reaching a certain "saturated" state (Fung, 1993; Humphrey, 2002). Another typical phenomenon known as permanent set is characterized by residual strains after unloading (Alastrué et al., 2008; Maher et al., 2012; García et al., 2013). Finally, there is the bond rupture and complete damage behavior resulting from fiber rupture and matrix disruption be previous to the fracture of the tissue which is not related to the Mullins effect (Noble et al., 2016). 


\begin{tabular}{|c|c|c|c|c|c|c|c|c|c|c|}
\hline Specimen & $\mu$ & $k_{1}$ & $k_{2}$ & $\theta$ & $\kappa$ & $\alpha_{m}$ & $\gamma_{m}$ & $\alpha_{f}$ & $\gamma_{f}$ & $R^{2}$ \\
\hline \multicolumn{11}{|c|}{ Whole artery } \\
\hline$I I$ & 0.0027 & 4.5969 & 56.5479 & 0.15 & 0.3248 & 10.0033 & 2.8425 & 57.5256 & 0.2052 & 0.8783 \\
\hline$I V$ & 0.0086 & 0.2483 & 14.8579 & 35.06 & 0.2744 & 55.8691 & 0.2267 & 10.2558 & 0.2116 & 0.8673 \\
\hline$V$ & 0.0165 & 0.1127 & 15.6244 & 0.00 & 0.0 & 256.3630 & 0.6196 & 9.2194 & 0.1738 & 0.7216 \\
\hline$V I$ & 0.0100 & 0.2932 & 0.3762 & 32.42 & 0.0345 & 6.5934 & 5.1020 & 140.5719 & 0.5187 & 0.9799 \\
\hline$V I I$ & 0.0100 & 6.0486 & 14.63 & 26.95 & 0.3256 & 99.3045 & 8.8237 & 100.0993 & 0.3098 & 0.4709 \\
\hline \multicolumn{11}{|l|}{ Intima } \\
\hline$I$ & 0.0075 & 1.2154 & 5.0033 & 0.63 & 0.2511 & 13.2331 & 0.0017 & 98.9175 & 0.9791 & 0.9628 \\
\hline$I I$ & 0.0130 & 3.3308 & 13.6472 & 28.87 & 0.2828 & 0.0010 & 0.0249 & 186.5920 & 0.2745 & 0.9216 \\
\hline III & 0.0084 & 0.0425 & 0.5960 & 0.00 & 0.0 & 194.9276 & 0.3765 & 20.0012 & 0.4432 & 0.7138 \\
\hline$I V$ & 0.0118 & 0.1087 & 1.9562 & 32.94 & 0.0505 & 47.7340 & 0.9933 & 99.0223 & 0.4500 & 0.6955 \\
\hline$V$ & 0.0100 & 2.8077 & 0.0010 & 34.16 & 0.0 & 159.9684 & 0.3568 & 0.0413 & 0.5101 & 0.8653 \\
\hline \multicolumn{11}{|l|}{ Media } \\
\hline$I$ & 0.0100 & 0.2897 & 3.3841 & 34.73 & 0.0 & 207.1045 & 0.3798 & 97.6807 & 0.6003 & 0.9206 \\
\hline$I I$ & 0.0098 & 1.4816 & 11.8609 & 12.66 & 0.2730 & 16.0621 & 0.3660 & 210.5620 & 0.2005 & 0.8803 \\
\hline$I I I$ & 0.0123 & 0.6484 & 2.7538 & 37.16 & 0.2495 & 116.1468 & 0.8372 & 185.4415 & 0.3648 & 0.5862 \\
\hline$I V$ & 0.0148 & 0.4260 & 4.5274 & 0.04 & 0.2676 & 44.9062 & 0.0020 & 97.2008 & 0.5440 & 0.9514 \\
\hline$V$ & 0.0105 & 1.3843 & 1.5508 & 52.79 & 0.2489 & 88.7689 & 0.2017 & 98.4999 & 13.9264 & 0.8334 \\
\hline$V I$ & 0.0129 & 0.3722 & 5.1647 & 26.82 & 0.0530 & 0.0100 & 0.2500 & 254.0749 & 0.2854 & 0.6116 \\
\hline$V I I$ & 0.0126 & 2.7596 & 1.0558 & 0.98 & 0.2541 & 281.9852 & 0.2801 & 499.0841 & 0.3357 & 0.7373 \\
\hline Mean & 0.0118 & 1.0517 & 4.3282 & 23.60 & 0.1923 & 107.8548 & 0.3310 & 206.0777 & 2.3224 & 0.7887 \\
\hline$S D$ & 0.0018 & 0.8953 & 3.6349 & 19.81 & 0.1146 & 103.7817 & 0.2562 & 143.6312 & 5.1188 & 0.1468 \\
\hline \multicolumn{11}{|l|}{ Adventitia } \\
\hline$I$ & 0.0095 & 3.4639 & 56.6768 & 4.45 & 0.3218 & 26.3130 & 0.4757 & 54.5822 & 0.2169 & 0.9099 \\
\hline II & 0.0074 & 16.6244 & 168.8492 & 67.52 & 0.3070 & 98.6211 & 0.0100 & 40.4556 & 0.2369 & 0.7067 \\
\hline
\end{tabular}

Table 3 Material constants obtained for the infrarenal abdominal aorta (IAA) curves for the phenomenological damage model. Constants $\mu$ and $k_{1}$ are in MPa, $\gamma_{m}, \gamma_{f}$ are in $\sqrt{M P a}, \theta$ in degrees, $k_{2}, \kappa, \alpha_{m}, \alpha_{f}$ and $R^{2}$ are dimensionless.

In this study, layer-specific experimental data relating to the rupture of swine aortas in uniaxial extension tests were collected and analyzed. Within the elastic range, the media layer seems to be the softest layer while the intima and adventitia exhibit considerable stiffness, independently of the position along the aorta. All tissue samples exhibit a pronounced nonlinear mechanical response and the anisotropic behavior for the elastic and rupture response is more marked in the abdominal aorta. Abdominal samples are stiffer than descending thoracic samples, with the most important differences corresponding to those between the descending thoracic and abdominal behaviors in the circumferential direction. It is generally agreed that aortic stiffness increases with increasing distance from the heart (Guo \& Kassab, 2003; Hang \& Fung, 1995; Kim et al., 2013; Peña et al., 2015, 2018). Our results are in agreement with the results presented by Peña et al. (Peña et al., 2015). These authors found statistically significant differences between the mechanical behavior of proximal and distal locations in circumferential directions, but not in the longitudinal direction because they tested the aorta at low stress-strains corresponding to the physiological regime. However, our results show that in the high loading domain the response in the longitudinal direction is also dependent on the position along the aorta, and the differences between DTA and IAA in the circumferential direction are less marked in the high loading domain than in the lower one. On the basis of these results, the extrapolation of the elastic mechanical properties from the physiological to the supra-physiological regime for characterizing the mechanical response of aorta would be inappropriate.

The circumferential complete and media samples displayed higher fracture stress $\left(\sigma_{r}\right)$ and the axial specimens higher fracture stretches $\left(\lambda_{r}\right)$ independently of the position; however, these differences are more marked for the abdominal samples. Similar results were presented by Noble et al. (Noble et al., 2016) who found greater fracture stress in the circumferential direction and greater fracture stretch in the longitudinal 


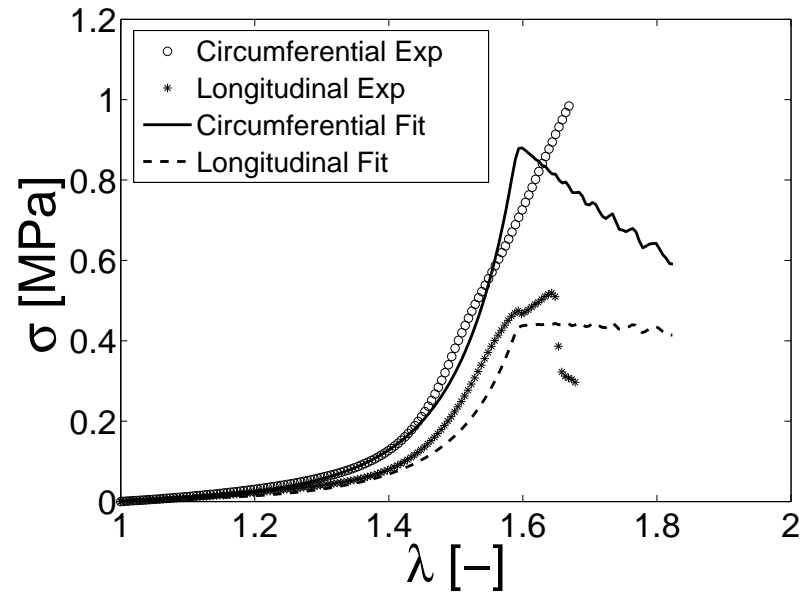

(a) Whole artery

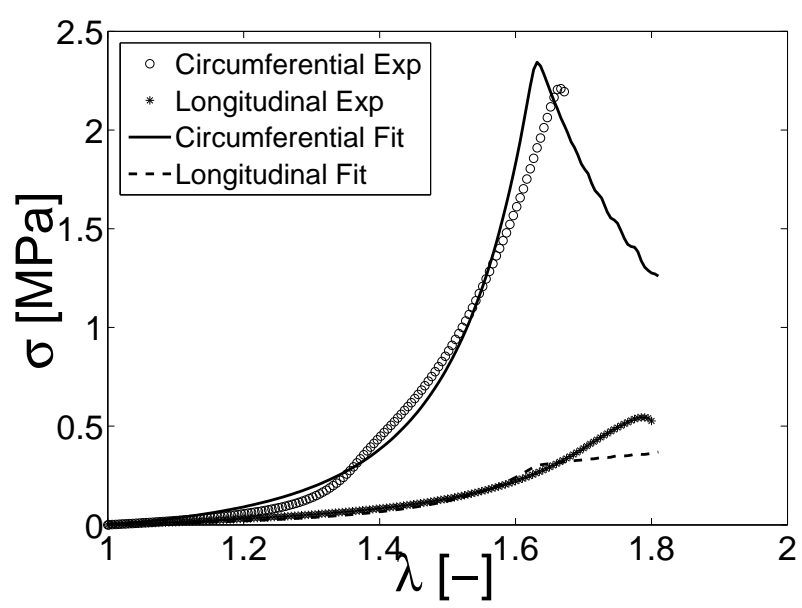

(c) Media

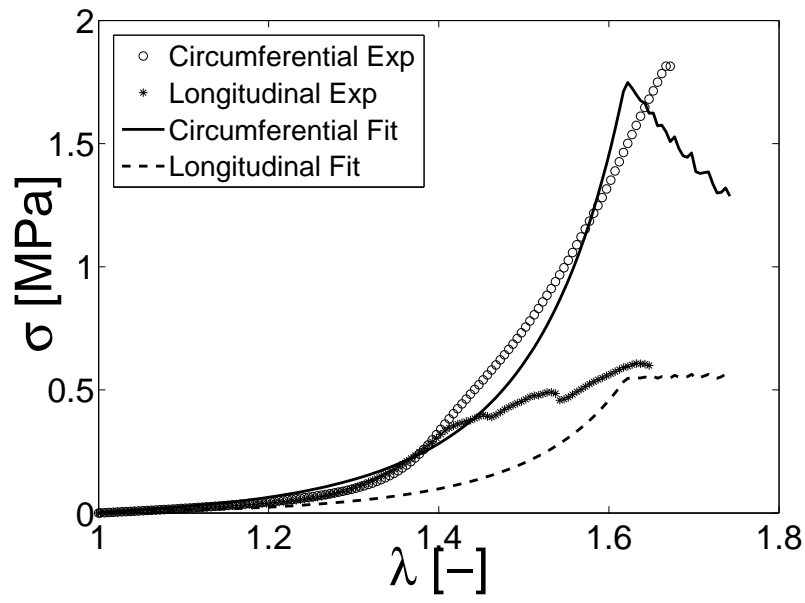

(b) Intima

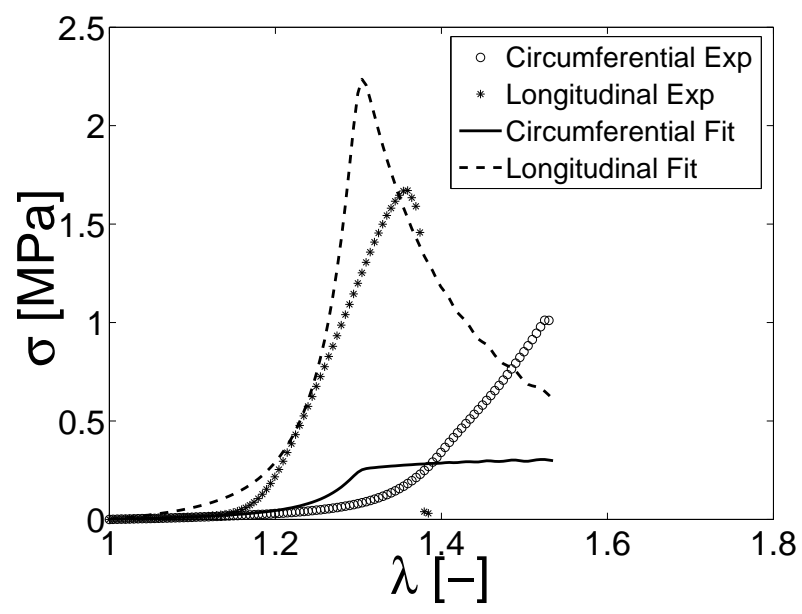

(d) Adventitia

Fig. 9 Representative DTA curves (III) and simulation results for (a) whole artery, (b) intima, (c) media and (d) adventitia with the microstructural damage model.

direction for the whole artery. The magnitude of the stress in the whole samples, where stress at fracture varied between $450-830 \mathrm{kPa}$ in the DTA samples and $1.38-2.60 \mathrm{MPa}$ in the IAA samples (Table 1), is similar to that reported in (Noble et al., 2016) and higher than in other publications (Holzapfel et al., 2005). Our results for the rupture properties for each of the separated layers demonstrated that the fracture stress is higher in the circumferential than in the longitudinal direction. This difference is relevant for the intima and media. Although the mechanics underlying aortic dissection are incompletely understood, due to the fact that the dissection process of blood flow delaminating the aorta is one of due to shear-induced injury, longitudinal failure properties would be more relevant ma- terial properties for analyzing the mechanics of Acute Aortic Dissection (AAD) (Rajagopal et al., 2019). For example, generally the fracture stress is higher in IAA compared to the equivalent intima and media DTA fracture stresses and ADD occurs more frequently in the ascending or descending aorta than in the abdominal one. However, we can not directly extend this conclusion to human ADD due to the fact that AAD are pathological whereas we have been tested healthy tissue. It is well-known that certain relationship can also be established between the microstructural composition of the arteries observed in the histological analysis and the elastic behavior of the tissue. However, the analysis of the fiber orientation and the constituent organization are outside the scope of this work. So, a 


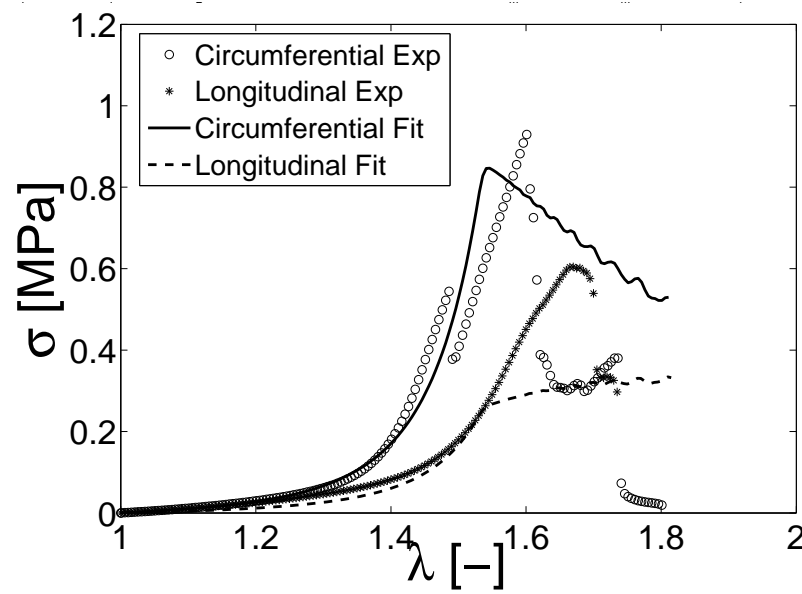

(a) Whole artery

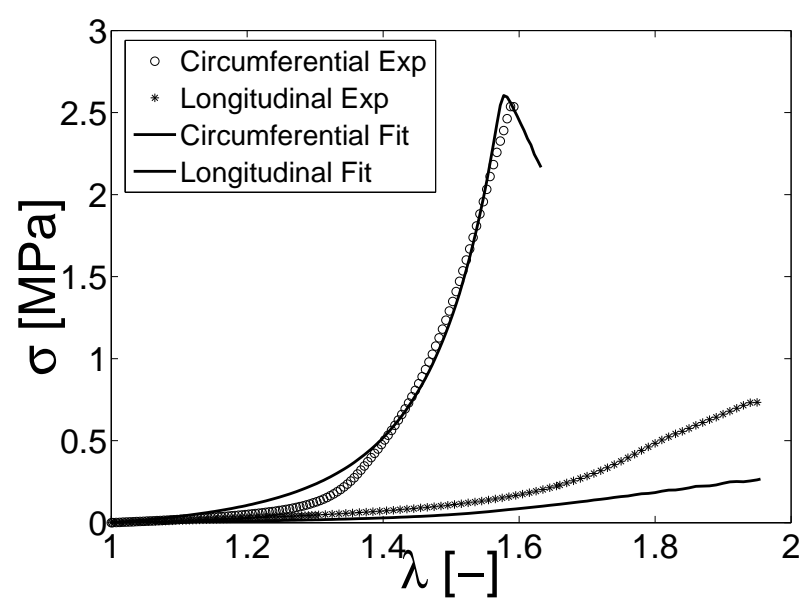

(c) Media

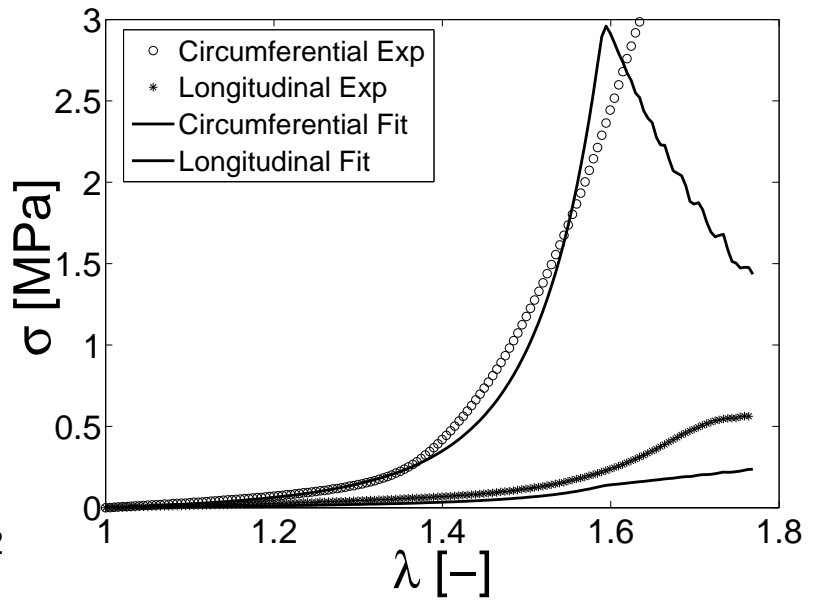

(b) Intima

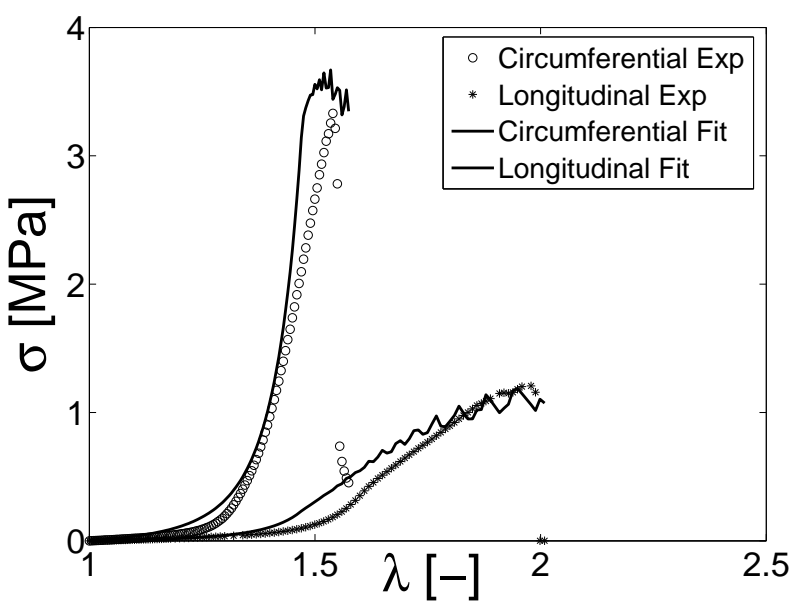

(d) Adventitia

Fig. 10 Representative IAA curves (IV) and simulation results for (a) whole artery, (b) intima, (c) media and (d) adventitia with the microstructural damage model.

further analysis of the histological data would be an interesting future analysis. Our findings are also analogous to those reported previously for coronary by Holzapfel et al. (Holzapfel et al., 2005). These authors found that ultimate tensile stresses of the media and intima are very similar and the strip samples from the adventitia showed about three times higher ultimate tensile stresses than samples from the related media and intima. Regarding the layer specific failure mechanical properties, similar results were obtained by Kim et al. (2012) for human ascending thoracic aortic aneurysms. They found no statistical differences between fracture stretches $\left(\lambda_{r}\right)$ for the circumferential or longitudinal directions. They also found that the longitudinal ultimate stress $\left(\sigma_{r}\right)$ was lower than circumferential ultimate stress for media and adventitia aortic aneurysms samples. Finally, they also found that the failure stress is much higher in the adventitia layer compared to that in the media layer as we can observe on Figure 5.

There are several constitutive models able to describe the failure of soft tissues in general, and arteries in particular. First, models based on Continuum Damage Mechanics (CDM), where a quantification of damage is performed by evaluating the reduction of the mechanical properties of the tissue using internal variables without a clear physical meaning can reproduce the softening behavior during unloading or reloading and rupture (Balzani et al., 2006; Calvo et al., 2007; Hokanson \& Yazdami, 1997; Peña, 2011c; Peña et al., 


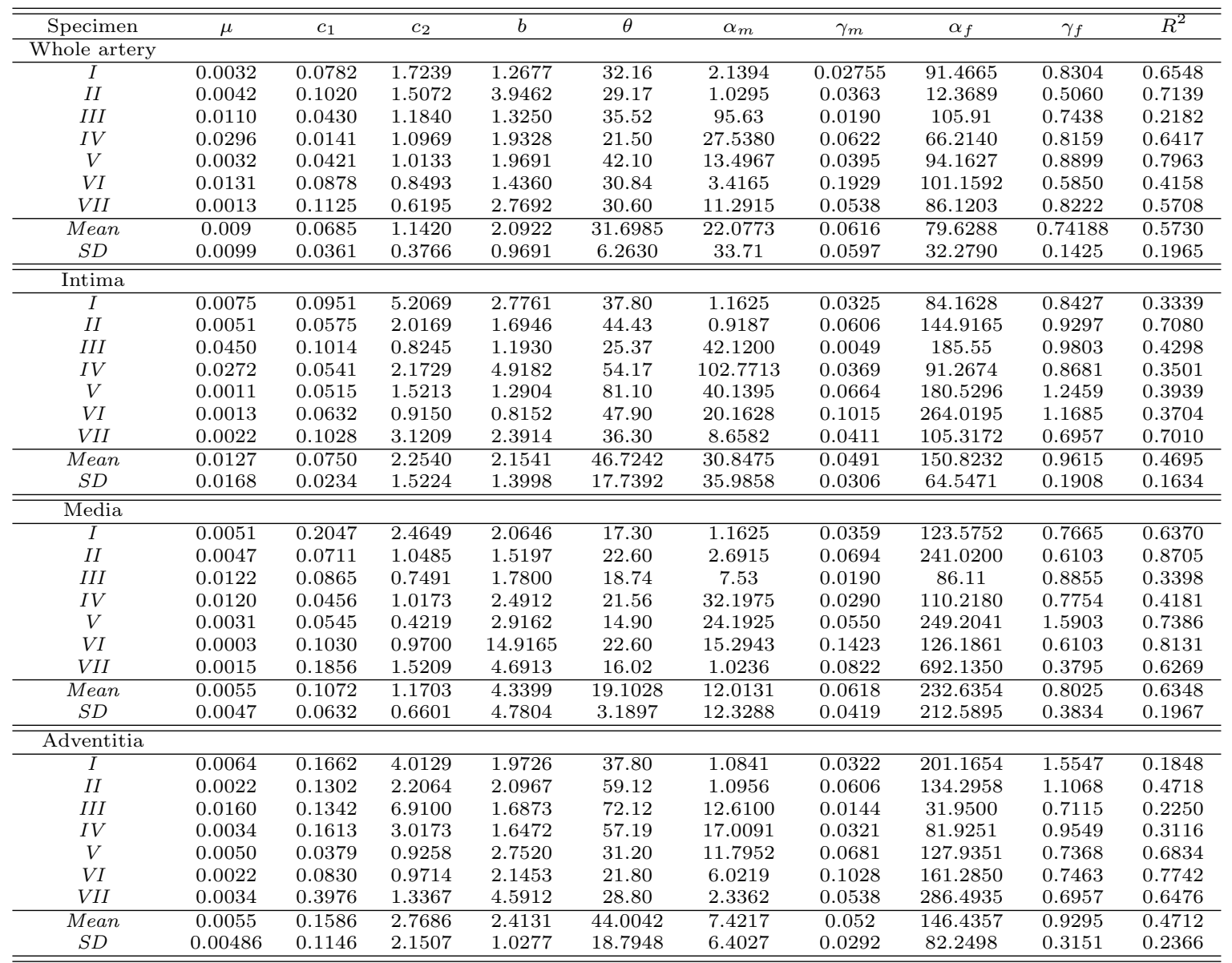

Table 4 Material constants obtained for the descending thoracic aorta (DTA) curves for the microstructural damage model. Constants $\mu$ and $c_{1}$ are in MPa, $\gamma_{m}, \gamma_{f}$ are in $\sqrt{M P a}, \theta$ in degrees, $c_{2}, b, \alpha_{m}, \alpha_{f}$ and $R^{2}$ are dimensionless.

2010, 2009). Models based on the theory of pseudoelasticity can represent damage-induced inelastic phenomena such as stress softening and permanent deformation (Peña, 2014; Peña \& Doblare, 2009; Weisbecker et al., 2012). Another option is using the softening hyperelasticity approach (Volokh, 2007b,a). Finally, fracture mechanics models can predict the rupture of soft biological tissues in computational simulations. These include the cohesive zone model (CZM) (Ferrara \& Pandolfi, 2010; Forsell \& Gasser, 2011; Hernández \& Peña, 2016), the extended finite element method (XFEM) (Gasser \& Holzapfel, 2006) and the crack phase-field model (CPFM) (Gültekin et al., 2016; Raina \& Miehe, 2016). For their simplicity and applicability, CDM phenomenological models are good candidates for modelling stress softening in biological soft tissues. In addition, the extension to microstructural models are based on internal variables is easily formulated (Sáez et al., 2012). For these reasons, CDM phenomenological and microstructural models are used in this work to reproduce the experimental data.

The CDM phenomenological damage model gives an excellent fit to the experimental data compared with the CDM microstructural damage model. It has the same number constitutive parameters, two parameters to account for damage in the matrix and another two parameters for the fibers. A comparison of the quality of the curve fits in the circumferential and axial directions suggests that the CDM phenomenological model is best suited for smooth, continuous breaking. The error measure $R^{2}>0.7$ is very close to one mainly for arterial layers and specimens. This indicates a very good correlation between the model and the experimental data for all arterial layers and specimens. The worst fit $R^{2}>0.407$ is always when the damage response is highly anisotropic and the maximum load in the circumferential and longitudinal directions are totally different. In these cases, the fitting curve in the longitudinal direction underestimates the stress due to the coupled character of the damage model. Due 


\begin{tabular}{|c|c|c|c|c|c|c|c|c|c|c|}
\hline Specimen & $\mu$ & $c_{1}$ & $c_{2}$ & $b$ & $\theta$ & $\alpha_{m}$ & $\gamma_{m}$ & $\alpha_{f}$ & $\gamma_{f}$ & $R^{2}$ \\
\hline \multicolumn{11}{|l|}{ Whole artery } \\
\hline$I$ & 0.0072 & 0.1489 & 4.1996 & 0.7516 & 21.30 & 1.2139 & 0.0682 & 103.4952 & 1.5547 & 0.6751 \\
\hline II & 0.0037 & 0.1150 & 5.9427 & 1.0924 & 40.10 & 18.4619 & 0.0459 & 361.3021 & 1.3123 & 0.6734 \\
\hline III & 0.0170 & 0.0415 & 0.9030 & 1.2175 & 10.00 & 16.41 & 0.01758 & 71.56 & 0.7905 & 0.4454 \\
\hline$I V$ & 0.0034 & 0.0313 & 1.6181 & 1.9432 & 33.19 & 17.0091 & 0.0321 & 81.9251 & 0.6070 & 0.5152 \\
\hline$V$ & 0.0011 & 0.9922 & 5.7918 & 5.1934 & 25.90 & 63.1960 & 0.0885 & 46.1952 & 0.6457 & 0.3977 \\
\hline$V I$ & 0.0013 & 0.1036 & 1.3971 & 2.1453 & 29.18 & 12.4195 & 0.0379 & 198.1645 & 1.1574 & 0.7406 \\
\hline$V I I$ & 0.0021 & 0.1492 & 6.5392 & 2.1755 & 40.10 & 1.6615 & 0.0686 & 151.2037 & 1.2017 & 0.5035 \\
\hline Mean & 0.0051 & 0.2260 & 3.7702 & 2.0741 & 28.5386 & 18.6246 & 0.0513 & 144.8351 & 1.0385 & 0.5644 \\
\hline$S D$ & 0.0056 & 0.3411 & 2.4201 & 1.4837 & 10.7426 & 20.9107 & 0.0248 & 108.4038 & 0.3615 & 0.1312 \\
\hline \multicolumn{11}{|l|}{ Intima } \\
\hline$I$ & 0.0036 & 0.1586 & 2.1971 & 2.5920 & 20.75 & 4.0295 & 0.0544 & 65.0200 & 0.8323 & 0.8087 \\
\hline II & 0.0051 & 0.1960 & 3.4943 & 3.0924 & 35.10 & 6.7150 & 0.0332 & 129.4185 & 0.9961 & 0.5636 \\
\hline III & 0.0192 & 0.0599 & 0.7903 & 4.6250 & 25.00 & 19.4100 & 0.0504 & 101.5660 & 0.8111 & 0.3074 \\
\hline$I V$ & 0.0131 & 0.0392 & 1.3173 & 2.8192 & 20.62 & 5.1942 & 0.0258 & 101.5754 & 0.7936 & 0.4317 \\
\hline$V$ & 0.1222 & 0.1242 & 0.6789 & 2.1934 & 72.50 & 2.2019 & 0.4541 & 119.4495 & 1.1685 & 0.7595 \\
\hline$V I$ & 0.0129 & 0.1711 & 0.9714 & 3.1315 & 22.30 & 6.0197 & 0.0427 & 267.2019 & 0.7147 & 0.6353 \\
\hline$V I I$ & 0.0048 & 0.2862 & 8.1184 & 4.6175 & 41.50 & 2.1658 & 0.0550 & 73.4941 & 1.3282 & 0.4385 \\
\hline Mean & 0.0258 & 0.1479 & 2.5097 & 3.2959 & 33.9671 & 6.5337 & 0.1022 & 122.5322 & 0.9492 & 0.5635 \\
\hline$S D$ & 0.0429 & 0.0839 & 2.6652 & 0.9591 & 18.7625 & 5.9442 & 0.1555 & 67.7955 & 0.2258 & 0.1836 \\
\hline \multicolumn{11}{|l|}{ Media } \\
\hline$I$ & 0.0038 & 0.1663 & 2.7164 & 5.1463 & 25.62 & 10.5160 & 0.0385 & 143.2189 & 0.6325 & 0.5467 \\
\hline$I I$ & 0.0040 & 0.1953 & 2.9420 & 3.7165 & 31.70 & 11.5492 & 0.0417 & 95.4028 & 0.7115 & 0.5459 \\
\hline$I I I$ & .0145 & 0.1040 & 1.0766 & 1.9105 & 34.50 & 11.8300 & 0.0685 & 45.1920 & 0.9360 & 0.5639 \\
\hline$I V$ & 0.0092 & 0.0640 & 1.0194 & 4.1849 & 22.19 & 3.9425 & 0.0164 & 105.7140 & 0.7245 & 0.5644 \\
\hline$V$ & 0.0162 & 0.5432 & 5.7918 & 3.1934 & 24.30 & 15.2019 & 0.4981 & 164.2940 & 0.6460 & 0.7170 \\
\hline$V I$ & 0.0022 & 0.3710 & 0.2571 & 4.2294 & 11.50 & 14.2197 & 0.0822 & 651.0920 & 0.4111 & 0.5316 \\
\hline$V I I$ & 0.0031 & 0.3246 & 3.6115 & 3.6175 & 24.10 & 6.4195 & 0.0601 & 157.3654 & 0.6008 & 0.5656 \\
\hline Mean & 0.0076 & 0.2526 & 2.4878 & 3.7141 & 24.8443 & 10.5255 & 0.1151 & 194.6113 & 0.6661 & 0.5764 \\
\hline$S D$ & 0.0058 & 0.1693 & 1.8957 & 1.0060 & 7.3802 & 4.0509 & 0.1703 & 205.5048 & 0.1577 & 0.0633 \\
\hline \multicolumn{11}{|l|}{ Adventitia } \\
\hline$I$ & 0.0027 & 0.0942 & 6.9450 & 0.8625 & 40.18 & 0.5160 & 0.0266 & 205.9125 & 1.5811 & 0.6283 \\
\hline II & 0.0067 & 0.1890 & 21.3419 & 3.2165 & 55.23 & 8.7152 & 0.0632 & 143.2985 & 1.3993 & 0.5965 \\
\hline$I I I$ & 0.0074 & 0.1068 & 4.6941 & 2.1933 & 24.60 & 19.1676 & 0.0306 & 75.1353 & 0.8412 & 0.6091 \\
\hline$I V$ & 0.0051 & 0.0802 & 2.5347 & 4.1749 & 31.15 & 5.1694 & 0.0247 & 161.0230 & 0.9866 & 0.7755 \\
\hline$V$ & 0.0462 & 0.4894 & 0.9332 & 2.6154 & 22.70 & 1.2956 & 0.6185 & 213.4195 & 2.1171 & 0.6716 \\
\hline$V I$ & 0.0139 & 0.5911 & 2.0135 & 3.9450 & 28.50 & 8.7502 & 0.0917 & 152.0946 & 0.7273 & 0.4363 \\
\hline$V I I$ & 0.0057 & 0.2658 & 2.9196 & 1.4765 & 42.40 & 10.1658 & 0.0870 & 61.2820 & 2.0871 & 0.6632 \\
\hline Mean & 0.0125 & 0.2595 & 5.9117 & 2.6406 & 34.9657 & 7.6828 & 0.1346 & 144.5951 & 1.3914 & 0.6258 \\
\hline$S D$ & 0.0152 & 0.2044 & 7.0826 & 1.2318 & 11.6000 & 6.3080 & 0.2152 & 58.5807 & 0.5707 & 0.1024 \\
\hline
\end{tabular}

Table 5 Material constants obtained for the infrarenal abdominal aorta (IAA) curves for the microstructural damage model. Constants $\mu$ and $c_{1}$ are in MPa, $\gamma_{m}, \gamma_{f}$ are in $\sqrt{M P a}, \theta$ in degrees, $c_{2}, b, \alpha_{m}, \alpha_{f}$ and $R^{2}$ are dimensionless.

to the phenomenological character of the model, the damage parameters $\left(\alpha_{m}, \gamma_{m}, \alpha_{f}, \gamma_{f}\right)$ present high dispersion (higher SD compare with Mean). For example, $\gamma_{f}$ that controls when the damage starts has values of $0.2753 \pm 0.2167,0.4018 \pm 0.3266,0.2969 \pm 0.1912$ and $0.3461 \pm 0.2249$ for whole, intima, media and adventitia of the DTA, respectively, and $0.2841 \pm 0.1187$, $0.4679 \pm 0.2428,2.3224 \pm 5.1188$ and $0.3467 \pm 0.1601$ for whole, intima, media and adventitia of the IAA.

The CDM microstructural damage model gives a questionable fit to the experimental data compared with the CDM phenomenological damage model. The error measure $R^{2}<0.87$ is far from one for some arterial layers and specimens. The worst fit is always when the elastic response is quasi-isotropic and the damage response is highly anisotropic, and the maximum load for the circumferential and longitudinal directions are totally different, as also occurs with the phenomenological model. In summary, samples which have a narrow damage zone indicating rapid damage and rupture have a worse fit than samples suffering a soft damage process identifiable by the smooth roundshaped damage zone. This fact is due to the discrete local integration character of the microstructural damage model. When one fiber associated to one direction of integration fails, other fibrils associated to another integration direction support the load causing a nonsmother stress response (Sáez et al., 2012). In contrast to the phenomenological model, the damage fibrils parameters $\left(\alpha_{f}, \gamma_{f}\right)$ have lower dispersion (lower SD compared with the Mean) due to the fact that these parameters represent the damage properties of the fibrils. They would be similar independently of the layers and positions, as can be seen in Tables 4 and 5 which show values of $0.74188 \pm 0.1425,0.9615 \pm 0.1908$, $0.8025 \pm 0.3834$ and $0.9295 \pm 0.3151$ for whole, intima, media and adventitia of the DTA, respectively, and $1.0385 \pm 0.3615,0.9492 \pm 0.2258,0.6661 \pm 0.1577$ and $1.3914 \pm 0.5707$ for whole, intima, media and adventitia of the IAA. Another advantage of microstructural models is they that can include physically motivated aspects obtained from histological analysis, polarized 
light microscopy (Schriefl et al., 2012) or other quantitative experimental techniques (Rezakhaniha et al., 2012).

There are few works that fit experimental data of of artery damage and rupture using CDM models. Only Noble et al. (Noble et al., 2016) fit their experimental data of healthy and treated porcine media thoracic aorta with collagenase, elastase and glutaraldehyde in order to simulate an aneurysm. The constitutive model used comprised the Gasser-OgdenHolzapfel model (Gasser et al., 2006) for elastic response and a continuum damage model for the failure. However these authors found that the damage parameters had to be different in the axial and circumferential directions in order to get good correlation results. In contrast, in this work we fitted the damage parameters at the same time for the circumferential and longitudinal direction. Fitting both directions separately allows better correlation results when the damage response is highly anisotropic and the maximum load for the circumferential an longitudinal directions are totally different. However, this methodology is not useful if we want to use computational models to analyze physiological and pathological aspects of arteries in terms of aortic dissection or aneurysmatic and atherosclerotic rupture, for example. It is important to remark that our model is able to predict similar correlation results using the same fiber parameters.

The findings of this study should be interpreted within the context of its limitations. Regarding the experimental analysis, a small number of tissue samples $(n=7)$ were investigated. Another experimental limitation is related to the use of simple tension tests for separated layers. It has previously been pointed out (Holzapfel et al., 2005; Peña et al., 2015) that a layer separation of arteries into their tissue components in young samples is difficult. It was impossible to separate large square samples for biaxial tests, so only uniaxial rectangular strips with a 5:1 lengthwidth ratio were used for the uniaxial tests. Another limitation of the study is that the structural integrity at the lateral edges of the strips is modified during the separation process. As pointed out by Holzapfel et al. (Holzapfel et al., 2005), collagenous fibers that are cut off during preparation may retract and inappropriate layer separation could occur. To check the correct anatomical separation of the arterial tissue into its three corresponding layers, a histological analysis using Hematoxiline-eosine stain was carried out and some strips had to be discarded during postprocessing as the layer separation process was not successful. Finally, all mechanical properties were reported un- der the assumption of homogeneity, which is clearly not the case for arteries.

With regard to material fitting, the CDM models used here do not take into account viscoelastic effects, softening due to Mullin's effect or permanent set. We use an experimental protocol with a single loading part until rupture, so we do not have experimental data to reproduce these effects. There is data in the literature suggesting that aortic tissue is highly compressible (Nolan \& McGarry, 2016). We do not have enough information to check this hypothesis, so we assume the arterial tissue as incompressible material (Carew et al., 1968). In addition, our CDM models consider only the passive mechanical response of aortic tissues. However, active response due to smooth muscle cells may also play an important role in damage accumulation (Weisbecker et al., 2012). Due to the phenomenological nature of the CDM phenomenological model, it is very difficult to associate the internal variables $D_{i}$ with microstructural changes or internal processes. Another limitation is related to the undetermined parameter fitting problem. The approach followed in this work to fit the experimental data is known to lead to a non-unique set of parameters able to reproduce the material behavior. For the microstructural model, this underdetermination would be a direct measurement of the micro-structural parameters, through experimental tests. For example, (Schriefl et al., 2012; Polzer et al., 2015; Sáez et al., 2016) performed measures with polarized light microscopy in order to obtain the concentration parameters of the fibers. Another limitation of the microstructural model is associated with the discrete integration performed that leads to a non-smooth response of the damage response of the microsphere and a mismatch between the statistical distribution of the concentration parameter and the uniform placing of the integration directions. In cases with highly concentrated distributions, many of the integration directions are not used in an optimal manner, since they correspond to directions without fibrils. Instead of the integration method suggested by Bazănt \& Oh (Bažant \& Oh, 1986) with 600 integration directions, we need to use a Lebedev quadrature rule (Lebedev \& Laikov, 1999) for 77st algebraic order of accuracy. For the microstructural model, the use of the von Mises ODF is a simplification of the measured fiber distribution. The use of another ODF, such as the Bingham distribution, could lead to a more flexible description of the fiber distribution (Alastrué et al., 2010; Gasser et al., 2012; Sáez et al., 2016). The waviness of collagen fibers was not included by means of a probability density function for the recruitment stretch at which the fiber starts to bear a load 
(Weisbecker et al., 2015). This effect could improve the fitting of the microstructural model, as pointed out by Hamedzadeh et al. (Hamedzadeh et al., 2018) in a work where they propose a recruitment and damage constitutive model for collagen fibers in soft biological tissues. Similarly to other published models (Hurschler et al., 1997), their model employs probability distribution functions in order to capture the progressive recruitment and damage of fibrils in a collagen fiber. We could also improve our model using a model that includes the interaction between collagen fibers and proteoglycan cross-linked collagen fibrils that undergo irreversible deformations, which here have been ignored (Gasser, 2011). Computational approaches such as finite element method have a key role to play in order to provide more realistic simulations of clinical interventions on supra-physiological scenarios where damage modelling should also be taken into account. For these cases the determination of the mechanical parameters is crucial to carry out simulations of realistic geometries under actual conditions. However, sometimes, the use of those constants in numerical models for complex load conditions does not guarantee that results obtained from a uniaxial test can be applied to a real state, as noted Duprey et al. (2016). They found statistical differences between bulge inflation and uniaxial tension experiments on $\sigma_{r}$ and $\lambda_{r}$ distributions of ascending thoracic aortic aneurysms. The observed that the ultimate stress in bulge inflation testing is similar to the axial ultimate stress and lower than circumferential ultimate stress in uniaxial tension tests. So, more realistic loading conditions on experimental tests would be needed.

\section{Conclusions}

There are three main findings in this study: first, in the high loading domain the response in the circumferential and longitudinal directions are dependently on the position along the aorta. The differences between the DTA and IAA in the circumferential direction are less marked in the high loading domain than in the lower one. This phenomenon is the contrary of the elastic regimen at the low loading domain, so the extrapolation of the elastic mechanical properties from the physiological to the supra-physiological regime for characterizing the mechanical response of the aorta would be inappropriate. Second, the fracture stress is higher in IAA compared to the equivalent DTA. This lower fracture stress in the media and adventitia in the longitudinal direction gives us a mechanical factor explaining the fact that Acute Aortic Dissection occurs more frequently in the thoracic aorta than in the abdominal aorta. Third, the CDM phenomenological damage model gives an excellent fit to the experimental data compared with the CDM microstructural damage model. Although the fitting results of the phenomenological model are better, the microstructural models can include physically motivated aspects obtained from experimental analysis and allow the integration of biochemical information of the architecture of arteries at micro level.

\section{Acknowledgments}

Financial support for this research was provided by the Spanish Ministry of Economy and Competitiveness through research project DPI2016-76630-C2-1-R; the Department of Industry and Innovation (Government of Aragon) through the research group Grant T24-17R (Fondo Social Europeo) and the Instituto de Salud Carlos III (ISCIII) through the CIBER initiative. The work was performed by the ICTS "NANBIOSIS" specifically by the Tissue \& Scaffold Characterization Unit (U13), of the CIBER in Bioengineering, Biomaterials \& Nanomedicne (CIBER-BBN at the University of Zaragoza). No conflicts of interest are declared.

\section{Disclosures}

No financial support from other organizations unless the cited in the acknowledgements section, nor personal relationships, nor people, nor organisms biased the development of this research work.

\section{References}

V. Alastrué, et al. (2009). 'Anisotropic micro-spherebased finite elasticity applied to blood vessel modelling'. J Mech Phys Solids 57:178-203.

V. Alastrué, et al. (2008). 'Experimental study and constitutive modelling of the passive mechanical properties of the ovine infrarenal vena cava tissue'. $J$ Biomech 41:3038-3045.

V. Alastrué, et al. (2010). 'On the use of bingham statistical distribution in microsphere-based constitutive models for arterial tissue'. Mech Res Commun 37:700-706.

D. Balzani, et al. (2006). 'Simulation of discontinuous damage incorporating residual stress in circumferentially overstretched atherosclerotic arteries'. Acta Biomater 2:609-618. 
J. G. Barra, et al. (1993). 'Assessment of Smooth Muscle Contribution to Descending Thoracic Aortic Elastic Mechanics in Conscious Dogs'. Circ Res 73:1040-1050.

P. Bažant \& B. H. Oh (1986). 'Efficient Numerical Integration on the Surface of a Sphere'. ZAMM-Z Angew Math Mech 66:37-49.

B. Calvo, et al. (2007). 'An uncoupled directional damage model for fibered biological soft tissues. Formulation and computational aspects'. Int J Numer Meth Engng 69:2036-2057.

T. E. Carew, et al. (1968). 'Compressibility of the arterial wall'. Circ Res 23:61-86.

H. Demiray (1972). 'A note on the elasticity of soft biological tissues'. J Biomech 5:309-311.

J. Diani, et al. (2009). 'A review on the Mullins effect'. Eur Polym J 45:601-612.

A. Duprey, et al. (2016). 'Biaxial rupture properties of ascending thoracic aortic aneurysms'. Acta Biomat 42:273-285.

A. E. Ehret \& M. Itskov (2009). 'Modeling of anisotropic softening phenomena: Application to soft biological tissues'. Int J Plasticity 25:901-919.

S. J. Eppell, et al. (2006). 'Nano measurements with micro-devices: mechanical properties of hydrated collagen fibrils'. J R Soc Interface 3:117-121.

A. Ferrara \& A. Pandolfi (2010). 'A numerical study of arterial media dissection processes'. Int $J$ Fract 166:21-33.

P. J. Flory (1961). 'Thermodynamic relations for high elastic materials'. Trans Faraday Soc 57:829-838.

C. Forsell \& T. C. Gasser (2011). 'Numerical simulation of the failure of ventricular tissue due to deeper penetration, the impact of constitutive properties'. $J$ Biomech 44:45-51.

Y. C. Fung (1993). Biomechanics. Mechanical properties of living tissues. Springer-Verlag, New York, second edn.

A. García, et al. (2013). 'Determination and Modeling of the Inelasticity Over the Length of the Porcine Carotid Artery'. ASME J Biomech Eng 135:031004-1.

T. C. Gasser (2011). 'An irreversible constitutive model for fibrous soft biological tissue: A 3-D microfiber approach with demonstrative application to abdominal aortic aneurysms'. Acta Biom 7:24572466.

T. C. Gasser, et al. (2012). 'Spatial orientation of collagen fibers in the abdominal aortic aneurysm's wall and its relation to wall mechanics'. Acta Biomat 8:3091-3103.

T. C. Gasser \& G. A. Holzapfel (2006). 'Modeling the propagation of arterial dissection'. Eur J Mech

\section{A/Solids 25:617-633.}

T. C. Gasser, et al. (2006). 'Hyperelastic modelling of arterial layers with distributed collagen fibre orientations'. J R Soc Interface 3:15-35.

O. Gültekin, et al. (2016). 'A phase-field approach to model fracture of arterial walls: theory and finite element analysis'. Comput Meth Appl Mech Eng 312:542-566.

X. Guo \& G. S. Kassab (2003). 'Variation of mechanical properties along the length of the aorta in C57bl/6 mice'. Am J Physiol Heart Circ Physiol 285:H2614-H2622.

A. Hamedzadeh, et al. (2018). 'On the constitutive modelling of recruitment and damage of collagen fibres in soft biological tissues'. Eur J Mech A/Solids 72:483-496.

H. C. Hang \& Y. C. Fung (1995). 'Longitudinal strain of canine and porcine aortas'. J Biomech 28:637641.

Q. Hernández \& E. Peña (2016). 'Failure properties of vena cava tissue due to deep penetration during filter insertion'. Biomech Model Mechanobiol 15:845-856.

J. Hokanson \& S. Yazdami (1997). 'A constitutive model of the artery with damage'. Mech Res Commun 24:151-159.

G. A. Holzapfel, et al. (2005). 'Determination of the layer-specific mechanical properties of human coronary arteries with non-atherosclerotic intimal thickening, and related constitutive modelling'. Am J Physiol Heart Circ Physiol 289:H2048-H2058.

G. A. Holzapfel, et al. (2000). 'A new constitutive framework for arterial wall mechanics and a comparative study of material models'. J Elasticity 61:1-48.

J. D. Humphrey (2002). Cardiovascular Solid Mechanics: Cells, Tissues, and Organs. Springer-Verlag, New York.

C. Hurschler, et al. (1997). 'A structurally based stress-stretch relationship for tendon and ligament'. ASME J Biomech Eng 119:392-399.

A. V. Kamenskiy, et al. (1998). 'Biaxial mechanical properties of the human thoracic and abdominal aorta, common carotid, subclavian, renal and common iliac arteries'. Biomech Model Mechanobiol 13:1341-1359.

J. Kim \& S. Baek (2011). 'Circumferential variations of mechanical behavior of the porcine thoracic aorta during inflation test'. J Biomech 44:1941-1947.

J. Kim, et al. (2013). 'Longitudinal differences in the mechanical properties of the thoracic aorta depend on circumferential regions'. J Biomed Mater Res Part A 101:1525-1529. 
J.-H. Kim, et al. (2012). 'Experimental characterization of rupture in human aortic aneurysms using a full-field measurement technique'. Biomechan Model Mechanobiol 11:841-853.

C. Lally, et al. (2004). 'Elastic Behavior of Porcine Coronary Artery Tissue under Uniaxial and Equibiaxial Tension'. Ann Biomed Eng 32:1355-1364.

V. I. Lebedev \& D. N. Laikov (1999). 'A quadrature formula for the sphere of the 131st algebraic order of accuracy'. Doklady Mathematics 59:477-481.

D. Li \& A. M. Robertson (2009). 'A Structural MultiMechanism Damage Model for Cerebral Arterial Tissue'. ASME J Biomech Eng 131:101013 1-8.

M. A. Lillie, et al. (2012). 'Contribution of elastin and collagen to the inflation response of the pig thoracic aorta: Assessing elastin's role in mechanical homeostasis'. J Biomech 45:2133-2141.

X. Lu, et al. (2003). 'Shear modulus of porcine coronary artery: contributions of media and adventitia'. Am J Physiol Heart Circ Physiol 285:H1966H1975.

E. Maher, et al. (2012). 'Site specific inelasticity of arterial tissue'. J Biomech 45:1393-1399.

D. W. Marquardt (1963). 'An algorithm for leastsquares estimation of nonlinear parameters'. Siam J Appl Math 11:431-441.

L. Mullins (1947). 'Effect of stretching on the properties of rubber'. Rubber Research 16:275-289.

C. Noble, et al. (2016). 'Creating a model of diseased artery damage and failure from healthy porcine aorta'. J Mech Behav Biomed 60:378-393.

D. R. Nolan \& J. P. McGarry (2016). 'On the Compressibility of Arterial Tissue'. Ann Biomed Eng 44:993-1007.

E. Peña (2011a). 'A rate dependent directional damage model for fibred materials. Application to soft biological tissues'. Comp Mech 48:407-420.

E. Peña (2011b). 'Damage functions of the internal variables for soft biological fibred tissues'. Mech Res Commun 38:610-615.

E. Peña (2011c). 'Prediction of the softening and damage effects with permanent set in fibrous biological materials'. J Mech Phys Solids 59:1808-1822.

E. Peña (2014). 'Computational aspects of the numerical modelling of softening, damage and permanent set in soft biological tissues'. Comput Struct 130:57-72.

E. Peña, et al. (2010). 'A constitutive formulation of vascular tissue mechanics including viscoelasticity and softening behaviour'. J Biomech 43:984-989.

E. Peña \& M. Doblare (2009). 'An anisotropic pseudoelastic approach for modelling Mullins effect in fibrous biological materials'. Mech Res Commun
36:784-790.

E. Peña, et al. (2009). 'On the Mullins effect and hysteresis of fibered biological materials: A comparison between continuous and discontinuous damage models'. Int J Solids Struct 46:1727-1735.

J. A. Peña, et al. (2018). 'Over length quantification of the multiaxial mechanical properties of the ascending, descending and abdominal aorta using Digital Image Correlation'. J Mech Behav Biomed 77:434445.

J. A. Peña, et al. (2015). 'Layer-specific residual deformations and uniaxial and biaxial mechanical properties of thoracic porcine aorta'. J Mech Behav Biomed 50:55-69.

D. M. Pierce, et al. (2015). 'Human thoracic and abdominal aortic aneurysmal tissues: Damage experiments, statistical analysis and constitutive modeling'. J Mech Behav Biomed 41:92-107.

S. Polzer, et al. (2015). 'Structure-based constitutive model can accurately predict planar biaxial properties of aortic wall tissue'. Acta Biomater 14:133145 .

A. Raina \& C. Miehe (2016). 'A phase-field model for fracture in biological tissues'. Biomech Model Mechanobiol 15:479-496.

K. Rajagopal, et al. (2019). 'The Mechanics of Acute Aortic Dissection: Measured Calculations and Calculated Measures'. J Thorac Cardiovasc Surg In press:- .

R. Rezakhaniha, et al. (2012). 'Experimental investigation of collagen waviness and orientation in the arterial adventitia using confocal laser scanning microscopy'. Biomech Model Mechanobiol 11:461-473.

J. F. Rodríguez, et al. (2006). 'A stochasticstructurally based three dimensional finite-strain damage model for fibrous soft tissue'. J Mech Phys Solids 54:564-886.

A. Schriefl, et al. (2012). 'Determination of the layerspecific distributed collagen fiber orientations in human thoracic and abdominal aortas and common iliac arteries'. J R Soc Interface 9:1275-1286.

P. Sáez, et al. (2012). 'Anisotropic microspherebased approach to damage in soft fibered tissue'. Biomechan Model Mechanobiol 11:595-608.

P. Sáez, et al. (2016). 'Microstructural quantification of collagen fiber orientations and its integration in constitutive modeling of the porcine carotid artery'. Acta Biomat 33:183-193.

F. H. Silver, et al. (2003). 'Mechanical Behavior of Vessel Wall: A Comparative Study of Aorta, Vena Cava, and Carotid Artery'. Ann Biomed Eng 31:793-803. 
J. C. Simo (1987). 'On a fully three-dimensional finite-strain viscoelastic damage model: Formulation and computational aspects'. Comput Methods Appl Mech Engrg 60:153-173.

D. P. Sokolis (2010). 'A passive strain-energy function for elastic and muscular arteries: correlation of material parameters with histological data'. Med Biol Eng Comput 48:507-518.

A. J. M. Spencer (1971). 'Theory of Invariants'. In Continuum Physics, pp. 239-253. Academic Press, New York.

K. Y. Volokh (2007a). 'Hyperelasticity with softening for modeling materials failure'. J Mech Phys Solids 55:2237-2264.

K. Y. Volokh (2007b). 'Prediction of arterial failure based on a microstructural bi-layer fiber-matrix model with softening'. J Biomech 41:447-453.

H. Weisbecker, et al. (2012). 'Layer-specific damage experiments and modeling of human thoracic and abdominal aortas with non-atherosclerotic intimal thickening'. J Mech Behav Biomed 12:93-106.

H. Weisbecker, et al. (2015). 'Constitutive modelling of arteries considering fibre recruitment and threedimensional fibre distribution'. $J R$ Soc Interface 12:20150111.

R. Wulandana \& A. M. Robertson (2005). 'An inelastic multi-mechanism constitutive equation for cerebral arterial tissue'. Biomech Model Mechanbiol 4:235-248.

S. Zeinali-Davarani, et al. (2013). 'Characterization of Biaxial Mechanical Behavior of Porcine Aorta under Gradual Elastin Degradation'. Ann Biomed Eng 41:1528-1538. 